\title{
openheart Effect of exercise therapy on established and emerging circulating biomarkers in patients with heart failure: a systematic review and meta-analysis
}

- Additional material is published online only. To view, please visit the journal online (http://dx.doi.org/10.1136/ openhrt-2018-000819).

To cite: Pearson MJ, King N, Smart NA. Effect of exercise therapy on established and emerging circulating biomarkers in patients with heart failure: a systematic review and metaanalysis. Open Heart 2018;5:e000819. doi:10.1136/ openhrt-2018-000819

Received 13 March 2018 Revised 4 May 2018 Accepted 24 May 2018

\section{Check for updates}

${ }^{1}$ School of Science and Technology, University of New England, Armidale, New South Wales, Australia

${ }^{2}$ School of Biomedical and Healthcare Sciences, University of Plymouth, Plymouth, UK

\section{Correspondence to}

Melissa J Pearson; mpears23@ myune.edu.au

\section{ABSTRACT}

Background Biomarkers are important in the diagnosis, risk stratification and management of patients with heart failure (HF). The established biomarkers of myocardial stretch, brain natriuretic peptide (BNP) and amino (N) portion of BNP (NT-proBNP) have been extensively studied, and early analyses have demonstrated response to exercise training. Several other biomarkers have been identified over the last decade and may provide valuable and complementary information which may guide treatment strategies, including exercise therapy. Methods A systematic search of PubMed, EMBASE and Cochrane Trials Register to 31 October 2017 was conducted for exercise-based rehabilitation trials in HF. Randomised and controlled trials that reported biomarkers, BNP, NT-proBNP, soluble ST2, galectin-3, mid-regional atrial natriuretic peptide, mid-regional adrenomedullin and copeptin, were included.

Results Forty-three studies were included in the systematic review, with 27 studies suitable for metaanalyses. Data pooling was only possible for NT-proBNP and BNP. Meta-analyses of conventional training studies demonstrated a statistically significant improvement in NT-proBNP (pmol/L); mean difference (MD) -32.80 (95\% $\mathrm{Cl}-56.19$ to -9.42 ), $\mathrm{p}=0.006$ and in BNP (pmol/L); MD $-17.17(95 \% \mathrm{Cl}-29.56$ to -4.78$), p=0.007$. Pooled data of non-conventional training failed to demonstrate any statistically significant improvements.

Conclusion Pooled data indicated a favourable effect of conventional exercise therapy on the established biomarkers, NT-proBNP and BNP; however, this was in contrast to a number of studies that could not be pooled. Limited evidence exists as to the effect of exercise training on emerging biomarkers.

\section{INTRODUCTION}

Heart failure (HF) is a complex syndrome resulting from multiple conditions and underlying disorders and continues to be a significant burden on the healthcare system. Over the past three decades, an increasing number of studies have provided evidence on a range of benefits of exercise training in patients with $\mathrm{HF}^{1-5}$ In patients with stable HF, exercise training is now a Class 1 recommendation in $\mathrm{HF}$ guidelines. ${ }^{67}$

\section{Key questions}

What is already known about this subject?

- Early reviews indicate that exercise training may improve brain natriuretic peptide (BNP) and amino (N) portion of BNP (NT-proBNP) in patients with heart failure (HF).

- A number of new trials have compared differen types of conventional and non-conventional modes of training on BNP and NT-proBNP, but the optimal exercise prescription for reducing HF biomarkers is unknown.

What does this study add?

- The review updates the evidence in regard to the effect of exercise training on the established HF biomarkers, BNP and NT-proBNP.

- Additionally, the response of a number of emerging biomarkers to exercise training has been investigated in patients with $\mathrm{HF}$.

- The pooled analysis of conventional exercise training confirms improvements in BNP and NT-proBNP but demonstrates only limited evidence for non-conventional training.

- Exercise training may also improve a number of other biomarkers representative of different pathophysiological pathways involved in HF progression.

How might this impact on clinical practice?

- The exercise prescription for patients with HF can be optimised to improve biomarker profile and hence prognosis, providing a valuable resource for both clinicians and patients.

Numerous pathways are involved in the development and progression of $\mathrm{HF}$, and the discovery of biomarkers has and will hopefully continue to enhance our understanding of the pathophysiology. ${ }^{9}$ Circulating biomarkers are important in the diagnosis, risk stratification and management of patients with HF. ${ }^{61011}$ HF biomarkers tend to be classified according to the associated pathophysiological processes. ${ }^{12} 13$ These include biomarkers of myocardial stretch, myocyte injury, fibrosis, matrix remodelling, inflammation, 
oxidative stress, neurohumoral activation and renal dysfunction. ${ }^{1012}{ }^{13}$ Some biomarkers may bridge several pathophysiological processes. Currently, brain (B-type) natriuretic peptide (BNP) and its more stable inert form, the amino (N terminal) portion (NT-proBNP), markers of myocardial stretch, are recognised as the gold standard diagnostic and prognostic biomarkers in HF. ${ }^{6} 711$

Over recent decades, the role of circulating biomarkers in HF has evolved, with the emergence of a number of novel biomarkers. ${ }^{12}$ Among these biomarkers, suppression of tumorigenicity 2 (ST2) and galectin-3 (Gal-3) have demonstrated prognostic value in $\mathrm{HF}^{14-17}$ and both are shown to be predictors of sudden cardiac death. ${ }^{18} 19$ In fact, the combination of the gold standard cardiac biomarkers of BNP/NT-proBNP with the newer biomarkers, such as soluble ST2 (sST2) and Gal-3, may improve risk stratification and prognosis. ${ }^{10}{ }^{11}$ Other emerging biomarkers, mid-regional atrial natriuretic peptide (MR-proANP), mid-regional adrenomedullin (MR-proADM) and copeptin (CT-proAVP), have also been shown to have prognostic value in $\mathrm{HF}^{9}{ }^{20}$

In addition to their diagnostic and prognostic utility, biomarker profiles may prove beneficial in guiding $\mathrm{HF}$ therapy and improving treatment strategies, ${ }^{10}$ including the identification of patients with HF that may respond to exercise training. ${ }^{21-23}$ A 2010 meta-analysis ${ }^{24}$ suggested that exercise training had a favourable effect on both BNP and NT-proBNP. The results of which were confirmed by a 2011 individual patient data (IPD) meta-analysis, with a $37.4 \%$ and $28.3 \%$ reduction in NT-proBNP and BNP, respectively. ${ }^{25}$ Furthermore, BNP and NT-proBNP changes are correlated with changes in peak oxygen consumption $\left(\mathrm{VO}_{2 \text { peak }}\right) \cdot{ }^{25}$

The aim of this systematic review and meta-analysis was first to update the previous reviews as a number of additional studies have investigated BNP and/or NT-proBNP after training interventions. Second, given the emergence of new biomarkers in HF trials, we intended to add to the current literature the inclusion of a selected number of emerging biomarkers. Furthermore, differing to previous analyses, we expanded our review to include additional modalities of exercise therapy due to their increasing utilisation in cardiac rehabilitation programmes and trials, which may provide alternatives for subgroups of patients with HF.

\section{METHODS}

\section{Search strategy}

Potential studies were identified by conducting systematic searches of PubMed, EMBASE, CINHAL and the Cochrane Library of Controlled Trials up until 31 October 2017. Searches included a mix of MeSH and free-text terms related to the key concepts of HF, exercise training and biomarkers. Additionally, systematic reviews, meta-analyses and reference lists of papers were hand searched for additional studies. One reviewer (MJP) conducted the search, and full articles were assessed for eligibility by two reviewers (MJP and NAS). A sample search strategy is presented in online supplementary files. Additional information was requested from five authors, with three responses.

\section{Study selection}

\section{Study type and participants}

Randomised controlled trials (RCTs) and controlled trials of exercise therapy in patients with HF aged 18 years or older were included. HF type (ie, preserved, moderately reduced and reduced ejection fraction) was not considered as an inclusion or exclusion criteria. Only studies in which the authors specifically reported a patient diagnosis of HF were included. Studies assessing intervention effect on acute or decompensated HF were excluded.

\section{Intervention}

Exercise therapy included both conventional training, defined as aerobic training (AT), resistance training (RT) and combined AT and RT, and non-conventional modes of therapy, defined as Yoga, Tai Chi, stretching and the physical therapies of functional electrical stimulation (FES) and inspiratory muscle training (IMT). Studies must have compared an exercise intervention to a usual care or education control group, with no formally prescribed exercise, and the duration of the exercise training must have been for a minimum of 4 weeks. Studies in which the participants had participated within a formal exercise rehabilitation programme within the last 6 months were excluded.

\section{Outcomes}

Studies were eligible to be included in the review if they reported one or more of the following outcomes in serum or plasma: BNP, NT-proBNP, cardiac troponin (cTnT), sST2, Gal-3, MR-proANP, MR-proADM and CT-proAVP.

\section{Exclusions}

Abstracts and non-English studies were excluded.

\section{Data extraction}

One reviewer (MJP) extracted the data. For each study, the following information was extracted: (1) author, year of publication and study design, (2) demographic and clinical characteristics, (3) exercise intervention characteristics, (4) mean, SD, P value and main findings in regard to biomarkers and (5) details of assessment methodology for biomarkers.

\section{Data synthesis}

Statistical analyses were performed using Revman V.5.3 (The Nordic Cochrane Centre, Copenhagen, Denmark). Individual meta-analyses were completed for continuous data by using the change in the mean and SD. Where the change in mean and SD was not reported, the change in mean was calculated by subtracting the preintervention mean from the postintervention mean, and Revman V.5.3 enabled calculations of SD using number of participants in each group, within or between group $p$ values or 
$95 \%$ CI. Where p values were not provided, the SD of the mean difference (MD) was calculated using the formula: $\mathrm{SD}=$ square root $\quad\left[\left(\mathrm{SD}_{\text {pretreatment }}\right)^{2}+\left(\mathrm{SD}_{\text {post-reatment }}\right)^{2}-(2 \mathrm{r}\right.$ $\left.\left.\times \mathrm{SD}_{\text {pretreatment }} \times \mathrm{SD}_{\text {post-reatment }}\right)\right]$, assuming a correlation coefficient $(r)=0.5$, which is considered a conservative estimate. ${ }^{26}$ Where data were not presented in text or tables and authors could not be reached, data presented in figures or reported in prior meta-analyses were extracted or accessed where possible.

Data were pooled for meta-analysis when two or more studies measured the same outcome and provided data in a format suitable for pooling. Where a study included multiple intervention groups and data were not provided for the combined intervention, data were entered separately for each group, and the sample size of the control group was divided by the number of intervention groups to eliminate overinflation of the sample size. A random-effects inverse variance was used with the effects to measure MD. We used a $5 \%$ level of significance and a 95\% CI to report change in outcome measures. Both BNP and NT-proBNP are commonly reported in SI units (pmol/L) or conventional units $(\mathrm{pg} / \mathrm{mL})$. Owing to large values associated with NT-proBNP, change data were converted from $\mathrm{pg} / \mathrm{mL}$ to $\mathrm{pmol} / \mathrm{L}$ for both NT-proBNP and BNP for presentation. Data were converted using the following factors: for NT-proBNP pmol $/ \mathrm{L}=\mathrm{pg} / \mathrm{mL} \times 0.118$ and BNP $\mathrm{pmol} / \mathrm{L}=\mathrm{pg} / \mathrm{mL} \times 0.289$.

For meta-analysis, we did not pool studies in which participants were clearly identified as only having heart failure with preserved ejection fraction (HFpEF), with other studies. We grouped studies for analysis according to conventional or non-conventional training modalities. For studies where the mean or SD of outcomes was not reported, but median, IQR or median and range were reported or where only a descriptive result was reported in regard to postintervention changes, a table and descriptive analysis are used.

Sensitivity analysis: In order to evaluate the influence of each study on the overall effect size, sensitivity analysis using the leave-one-out approach was conducted. Where SD was imputed, additional analyses were also carried out with different values for the correlation coefficient $(\mathrm{r}=0.75$ and 0.25$)$ to determine whether the overall results of the analyses were robust to the use of imputed correlation coefficients.

\section{Heterogeneity and publication bias}

Heterogeneity was quantified using the $\mathrm{I}^{2}$ test. ${ }^{27}$ Values range from $0 \%$ (homogeneity) to 100\% (high heterogeneity). ${ }^{27}$ Visual inspection of funnel plots ${ }^{28}$ assessed risk of publication bias.

\section{Study quality}

Study quality was assessed using the Tool for the Assessment of Study Quality and Reporting in Exercise $\left(\right.$ TESTEX) ${ }^{29}$ by two authors (MJP and NK). In case of discrepancies, a third author (NAS) was consulted.

\section{RESULTS}

The initial search generated a total of 3419 articles. After removal of duplicates and exclusion of articles based on abstract and title, 77 full-text articles remained for screening. Full screening resulted in 43 articles meeting the stated inclusion criteria (figure 1, Preferred Reporting Items for Systematic Reviews and Meta-Analyses statement), of which 27 studies were included in meta-analyses. Details of full-text articles reviewed but excluded are provided, with reasons, in online supplementary table S1.

\section{Study and participant characteristics}

A general description of included studies is provided in table 1 . Of the 43 included studies, two ${ }^{30} 31$ studies were from the same trial but provided different biomarker information, and two ${ }^{32} 33$ studies contained an overlap of some participants, and data were combined into one dataset for meta-analysis to eliminate data overlap. Four $^{22} 34-36$ of the studies were controlled but not randomised, one ${ }^{37}$ study randomised

participants between exercise intervention groups, but the control group was not randomised, one ${ }^{38}$ study was a retrospective analysis and all remaining studies were RCTs. Seven studies, ${ }^{30} 31{ }^{39-43}$ representing six trials, included participants with a mean left ventricular ejection fraction (LVEF) $>50 \%$, one ${ }^{43}$ of which also included participants with LVEF $<50 \%$. Thirty-six trials included participants with mean LVEF $<50 \%$, and the mean LVEF of at least three ${ }^{44-46}$ studies indicates the inclusion of participants with a range of ejection fractions, reduced, mid-range and/or preserved ejection fraction. Baseline NT-proBNP and BNP levels are provided in online supplementary table S2.

\section{Intervention details}

A detailed description of the interventions can be found in online supplementary table S3. Thirty-four studies used conventional exercise training, eight studies used non-conventional exercise training or therapy and one study combined non-conventional and conventional training. Intervention duration ranged from 4 weeks to 9 months.

\section{Biomarker assessment}

Biomarker assay details are provided in online supplementary table S4.

\section{Outcome measures}

\section{Amino (N) portion of BNP}

Twenty studies reported on NT-proBNP. Two studies $^{32} 33$ contained an overlap of some participants; to avoid possible duplication of data, these studies are represented as one dataset in the meta-analysis.

\section{Meta-analysis}

Overall, exercise demonstrated a statistically significant improvement in NT-proBNP (pmol/L); MD -47.83 (95\% CI -77.23 to -18.43 ), $\mathrm{p}=0.001$ (figure 2 ). 


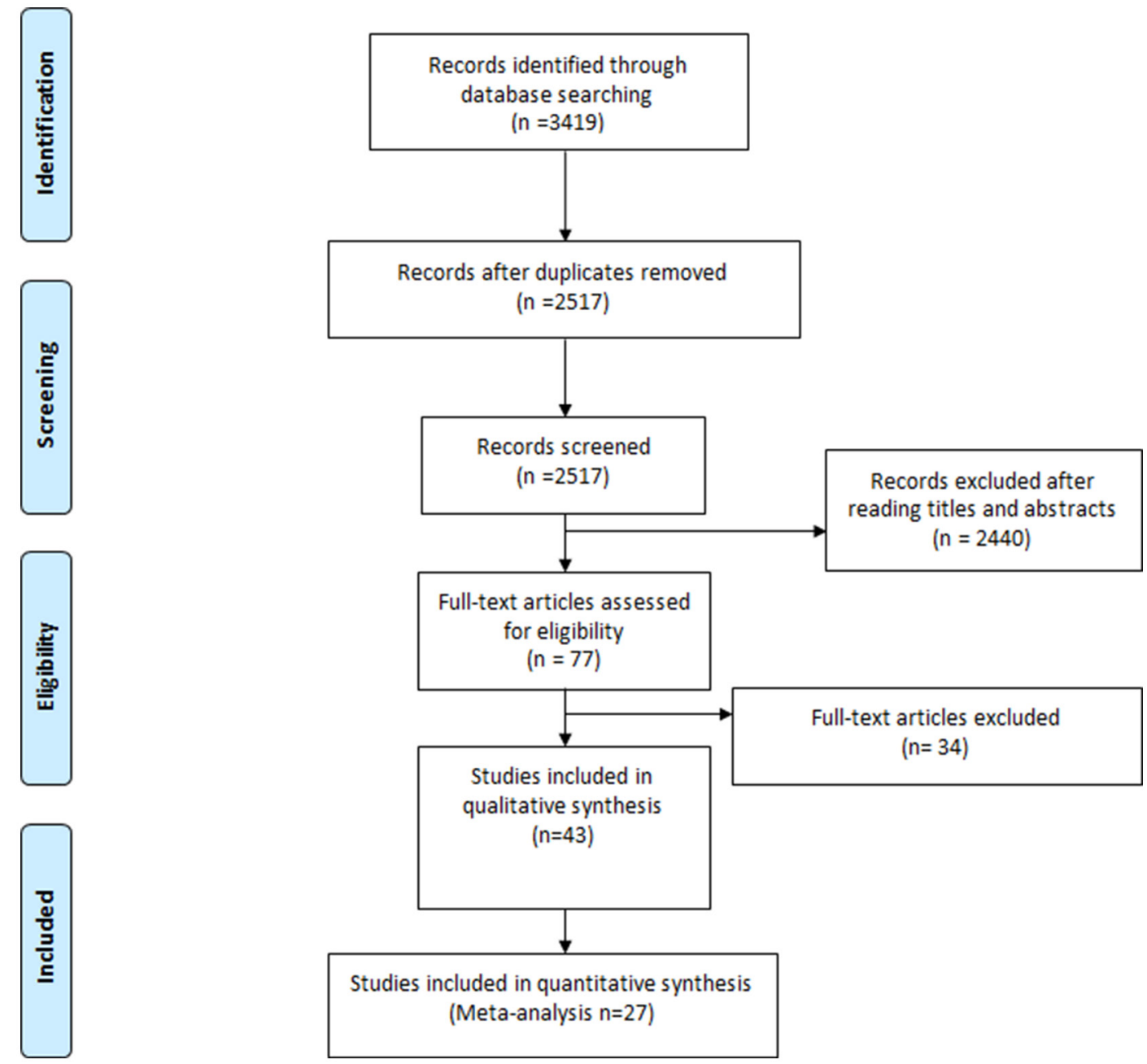

Figure 1 PRISMA flow diagram. PRISMA, Preferred Reporting Items for Systematic Reviews and Meta-Analyses.

\section{Conventional training}

Pooled data from 10 studies $^{32} 33353743$ 47-52 (14 intervention groups, 315 exercise participants and 212 controls) demonstrated a statistically significant improvement in favour of exercise, on NT-proBNP (pmol/L); MD -32.80 (95\% CI -56.19 to -9.42 ), $\mathrm{p}=0.006$ (figure 2 ). Removal of the two intervention groups from one ${ }^{43}$ study, that included patients with a mean ejection fraction of $50 \%$, improved the MD and statistical significance; MD -54.62 (95\% CI -74.36 to -34.87 ) pmol/L, $\mathrm{p}<0.00001$ (online supplementary table S5). Apart from the study by Aksoy $e t$ $a l,{ }^{43}$, sensitivity analysis using the leave-one-out approach revealed that the results remained relatively stable (figure 3). Sensitivity analyses conducted for different correlation coefficients for SD imputation did not result in any significant variance in overall results.

An additional six ${ }^{303453-55}$ studies (table 2 ) could not be pooled due to differences in data reporting. Five studies presented data as median (IQR) or median (range), and one ${ }^{30}$ study only included patients with HFpEF. Two studies $^{34}$ reported preintervention to postintervention NT-proBNP changes in exercise participants, but only one study reported a significant difference compared with control participants.
Non-conventional training

Pooled data from two ${ }^{45} 56$ studies $(55$ exercise participants and 59 controls) failed to demonstrate a statistically significant improvement in NT-proBNP ( $\mathrm{pmol} / \mathrm{L})$; MD -157.47 (95\% CI -327.64 to 12.70 ), $\mathrm{p}=0.07$ (figure 2 ). Notably, the large size of the improvement was due to the inclusion of one study ${ }^{45}$ (figure 4). One ${ }^{42}$ additional study, in patients with HFpEF, not pooled, failed to demonstrate any significant change (table 2 ).

\section{Brain natriuretic peptide}

Twenty-two studies reported on BNP. Two ${ }^{32} 33$ studies contained an overlap of some participants; to avoid duplication of data, these studies are represented as one dataset in the meta-analysis.

\section{Meta-analysis}

Overall, exercise demonstrated a statistically significant improvement in BNP (pmol/L); MD - 15.02 (95\% CI -25.06 to -4.99 ), $\mathrm{p}=0.003$ (figure 5 ).

Conventional training

Pooled data from 11 studies 32333846 57-64 (12 intervention groups, 268 exercise participants and 192 controls) 
Table 1 Overview of studies included in the review

\begin{tabular}{|c|c|c|c|}
\hline Study & Design & Participant characteristics & Intervention \\
\hline Ahmad et $a l^{53}$ & RCT & $\begin{array}{l}n=928 \text { analysed, biomarker substudy HF ACTION Trial } \\
E: ~ n=477\left(68 \% \text { male), } 59(51-68) \text { years, LVEF } 25 \%(20-30)^{*}\right. \\
\text { C: } n=451 \text { (73\% male), } 59(51-68) \text { years, LVEF } 25 \%(20-31) \\
\text { NYHA Class II-IV (<1\% IV) }\end{array}$ & $\begin{array}{l}3 \text { months } \\
\text { Aerobic }\end{array}$ \\
\hline Aksoy et al ${ }^{43}$ & $\mathrm{RCT}$ & $\begin{array}{l}n=57 \text { randomised, } n=45 \text { analysed } \\
\text { E1: } n=15(87 \% \text { male), } 64 \pm 9 \text { years, LVEF } 50 \% \pm 7 \% \\
\text { E2: } n=15(87 \% \text { male), } 60 \pm 7 \text { years, LVEF } 52 \% \pm 5 \% \\
\text { C: } n=15 \text { ( } 87 \% \text { male), } 58 \pm 11 \text { years, LVEF } 52 \% \pm 6 \% \\
\text { NYHA Class II-III }\end{array}$ & $\begin{array}{l}10 \text { weeks } \\
\text { Aerobic (E1: IAE, E2: CAE) }\end{array}$ \\
\hline Antonicelli et al ${ }^{44}$ & $\mathrm{RCT}$ & $\begin{array}{l}n=343 \text { randomised, } n=313 \text { completed } 6 \text { months } \\
E: n=170(61 \% \text { male), } 76 \pm 5 \text { years, LVEF } 48 \% \pm 13 \% \\
\text { C: } n=173(53 \% \text { male), } 78 \pm 6 \text { years, LVEF } 49 \% \pm 13 \% \\
\text { NYHA Class } \geq 2\end{array}$ & $\begin{array}{l}6 \text { months } \\
\text { Aerobic }\end{array}$ \\
\hline Billebeau et $a l^{22}$ & $\begin{array}{l}\text { Non-RCT } \\
\text { Cohort with control } \\
\text { group }\end{array}$ & $\begin{array}{l}n=131 \text { enrolled } \\
E: ~ n=107\left(86 \% \text { male), } 59(52-66) \text { years, LVEF } 30 \%(25-39)^{*}\right. \\
\text { C: } n=24(79 \% \text { male), } 63(53-72) \text { years, LVEF } 35 \%(30-40) \\
\text { NYHA Class II-IV }\end{array}$ & $\begin{array}{l}4-6 \text { months } \\
\text { Aerobic }\end{array}$ \\
\hline Brubaker et $a l^{65}$ & $\mathrm{RCT}$ & $\begin{array}{l}n=59 \text { randomised, } n=44 \text { analysed } \\
E: ~ n=30(63 \% \text { male), } 70 \pm 5 \text { years, LVEF } 32 \% \pm 9 \% \\
\text { C: } n=29(69 \% \text { male), } 70 \pm 6 \text { years, LVEF } 30 \% \pm 9 \% \\
\text { NYHA Class II-IV ( } n=1 \text { Class IV) }\end{array}$ & $\begin{array}{l}16 \text { weeks } \\
\text { Aerobic }\end{array}$ \\
\hline Butterfield et $a l^{58}$ & $\mathrm{RCT}$ & $\begin{array}{l}n=19 \text { randomised, } n=17 \text { analysed } \\
E: n=11(82 \% \text { male), } 66 \pm 10 \text { years, LVEF } 34 \% \pm 11 \% \\
\text { C: } n=6(50 \% \text { male), } 75 \pm 12 \text { years, LVEF } 35 \% \pm 14 \% \\
\text { NYHA Class } \|- \text {-III }\end{array}$ & $\begin{array}{l}12 \text { weeks } \\
\text { Combined }\end{array}$ \\
\hline Delagardelle et $\left.a\right|^{37}$ & RCT/non-RCT† & $\begin{array}{l}n=60 \text { randomised and analysed } \\
E: n=45(84 \% \text { male), } 59 \pm 6 \text { years, LVEF } 24 \% \pm 5 \% \\
\text { C: } n=15 \text { ( } 87 \% \text { male), } 56 \pm 8 \text { years, LVEF } 25 \% \pm 6 \% \\
\text { NYHA Class II }\end{array}$ & $\begin{array}{l}\sim 13.3 \text { weeks Combined, } \\
\text { aerobic or strength }\end{array}$ \\
\hline $\begin{array}{l}\text { Edelmann et } a^{30} \\
\text { Ex-DHF pilot study }\end{array}$ & $\mathrm{RCT}$ & $\begin{array}{l}n=67 \text { randomised, } n=64 \text { analysed } \\
E: ~ n=44(45 \% \text { male), } 64 \pm 8 \text { years, LVEF } 68 \% \pm 7 \% \\
\text { C: } n=20 \text { ( } 40 \% \text { male), } 65 \pm 6 \text { years, LVEF } 67 \% \pm 7 \% \\
\text { NYHA Class II and III }\end{array}$ & $\begin{array}{l}12 \text { weeks } \\
\text { Combined }\end{array}$ \\
\hline Eleuteri et $\left.a\right|^{54}$ & $\mathrm{RCT}$ & $\begin{array}{l}n=21 \text { randomised and analysed } \\
E: n=11(100 \% \text { male), } 66 \pm 2 \text { years, LVEF } 28 \% \pm 2 \% \\
\text { C: } n=10(100 \% \text { male), } 63 \pm 2 \text { years, LVEF } 30 \% \pm 2 \% \\
\text { NYHA Class II }\end{array}$ & $\begin{array}{l}3 \text { months } \\
\text { Aerobic }\end{array}$ \\
\hline Fernandes-Silva et $a l^{23}$ & $\mathrm{RCT}$ & $\begin{array}{l}n=52 \text { randomised, } n=40 \text { analysed } \\
E: n=28 \text { ( } 50 \% \text { male), } 51 \pm 7 \text { years, LVEF } 30 \% \pm 6 \% \\
\text { C: } n=16 \text { ( } 62 \% \text { male), } 48 \pm 7 \text { years, LVEF } 29 \% \pm 7 \% \\
\text { NYHA Class I-III }\end{array}$ & $\begin{array}{l}12 \text { weeks } \\
\text { Aerobic }\end{array}$ \\
\hline
\end{tabular}




\begin{tabular}{|c|c|c|c|}
\hline Study & Design & Participant characteristics & Intervention \\
\hline $\mathrm{Fu}(2013)^{57}$ & $\mathrm{RCT}$ & $\begin{array}{l}n=45 \text { randomised, } n=40 \text { analysed } \\
\text { E1: } n=15(67 \% \text { male), } 68 \% \pm 5 \% \text {, LVEF } 38 \% \pm 4 \% \\
\text { E2: } n=15(60 \% \text { male), } 66 \pm 2 \text { years, LVEF } 39 \% \pm 5 \% \\
\text { C: } n=15(67 \% \text { male), } 68 \pm 3 \text { years, LVEF } 38 \% \pm 4 \% \\
\text { NYHA Class } \|-1 I I\end{array}$ & $\begin{array}{l}12 \text { weeks } \\
\text { Aerobic (E1: AIT, E2: MCT) }\end{array}$ \\
\hline Guazzi et $a l^{49}$ & $\mathrm{RCT}$ & $\begin{array}{l}n=26 \text { randomised and analysed } \\
E: n=18, C: n=8,68 \pm 6 \text { years, LVEF } 37 \% \pm 5 \% \\
\text { NYHA Class } \|-1 I\end{array}$ & $\begin{array}{l}24 \text { weeks } \\
\text { Aerobic }\end{array}$ \\
\hline Karavidas et al ${ }^{66}$ & $\mathrm{RCT}$ & $\begin{array}{l}n=30 \text { randomised and analysed } \\
E: n=20 \text { ( } 80 \% \text { male), } 62 \pm 12 \text { years, LVEF } 28 \% \pm 7 \% \\
\text { C: } n=10 \text { ( } 80 \% \text { male), } 64 \pm 8 \text { years, LVEF } 27 \% \pm 5 \% \\
\text { NYHA Class II-III }\end{array}$ & $\begin{array}{l}6 \text { weeks } \\
\text { FES }\end{array}$ \\
\hline Karavidas et $\left.a\right|^{41}$ & RCT & $\begin{array}{l}n=30 \text { randomised and analysed } \\
E: n=15(60 \% \text { male), } 69 \pm 9 \text { years, LVEF } 64 \% \pm 8 \% \\
\text { C: } n=15(60 \% \text { male), } 69 \pm 8 \text { years, LVEF } 63 \% \pm 5 \% \\
\text { NYHA Class II-III }\end{array}$ & $\begin{array}{l}6 \text { weeks } \\
\text { FES }\end{array}$ \\
\hline Kato et $a l^{67}$ & $\mathrm{RCT}$ & $\begin{array}{l}n=50 \text { randomised and analysed } \\
E: \mathrm{n}=25(80 \% \text { male), } 70 \pm 11 \text { years, LVEF } 28 \% \pm 9 \% \\
\text { C: } n=25 \text { (76\% male), } 70 \pm 8 \text { years, LVEF } 29 \% \pm 9 \% \\
\text { NYHA Class II-IV }\end{array}$ & $\begin{array}{l}4 \text { weeks } \\
\text { Stretching }\end{array}$ \\
\hline Kitzman et $a l^{40}$ & $\mathrm{RCT}$ & $\begin{array}{l}n=53 \text { randomised, } n=46 \text { completed } \\
E: n=26(17 \% \text { male), } 70 \pm 6 \text { years, LVEF } 61 \% \pm 5 \% \\
\text { C: } n=27 \text { ( } 9 \% \text { male), } 69 \pm 5 \text { years, LVEF } 60 \% \pm 10 \% \\
\text { NYHA Class II-III }\end{array}$ & $\begin{array}{l}16 \text { weeks } \\
\text { Aerobic }\end{array}$ \\
\hline Kitzman et $a l^{39}$ & $\mathrm{RCT}$ & $\begin{array}{l}n=51 \text { randomised } \neq \\
E: n=26(19 \% \text { male), } 68 \pm 6 \text { years, LVEF } 61 \% \pm 6 \% \\
\text { C: } n=25(20 \% \text { male), } 66 \% \pm 5 \% \text {, LVEF } 63 \% \pm 6 \% \\
\text { NYHA Class } \|- \text {-III }\end{array}$ & $\begin{array}{l}20 \text { weeks } \\
\text { Aerobic }\end{array}$ \\
\hline Kobayashi et $a^{61}$ & RCT & $\begin{array}{l}n=28 \text { randomised and analysed } \\
E: n=14(86 \% \text { male), } 55 \pm 2 \text { years, LVEF } 29 \% \pm 2 \% \\
\text { C: } n=14 \text { ( } 57 \% \text { male), } 62 \pm 2 \text { years, LVEF } 33 \% \pm 2 \% \\
\text { NYHA Class II and III }\end{array}$ & $\begin{array}{l}12 \text { weeks } \\
\text { Aerobic }\end{array}$ \\
\hline Krishna et $a l^{45}$ & $\mathrm{RCT}$ & $\begin{array}{l}n=130 \text { randomised, } n=92 \text { analysed } \\
E: n=44(73 \% \text { male), } 49 \pm 6 \text { years, LVEF } 39 \% \pm 5 \% \\
\text { C: } n=48 \text { ( } 67 \% \text { male), } 50 \pm 5 \text { years, LVEF } 40 \% \pm 5 \% \\
\text { NYHA Class I- }-1\end{array}$ & $\begin{array}{l}12 \text { weeks } \\
\text { Yoga }\end{array}$ \\
\hline Malfatto et $a l^{60}$ & RCT & $\begin{array}{l}n=54 \text { randomised and analysed } \\
E: ~ n=27(70 \% \text { male), } 65 \pm 11 \text { years, LVEF } 31 \% \pm 6 \% \text {, } \\
\text { C: } n=27 \text { ( } 74 \% \text { male), } 67 \pm 9 \text { years, LVEF } 33 \% \pm 6 \% \text {, } \\
\text { NYHA Class I and } \|\end{array}$ & $\begin{array}{l}12 \text { weeks } \\
\text { Aerobic }\end{array}$ \\
\hline
\end{tabular}




\begin{tabular}{|c|c|c|c|}
\hline Study & Design & Participant characteristics & Intervention \\
\hline Marco et $a l^{56}$ & $\mathrm{RCT}$ & $\begin{array}{l}n=22 \text { randomised and analysed } \\
E: n=11(64 \% \text { male), } 69 \pm 9 \text { years, LVEF } 38 \% \pm 16 \% \\
\text { C: } n=11 \text { ( } 91 \% \text { male), } 70 \pm 11 \text { years, LVEF } 36 \% \pm 17 \% \\
\text { NYHA Class } \|- \text { III }\end{array}$ & $\begin{array}{l}4 \text { weeks } \\
\text { IMT }\end{array}$ \\
\hline Meyer et $a l^{50}$ & $\mathrm{RCT}$ & $\begin{array}{l}n=42 \text { randomised and analysed } \\
E: n=19(79 \% \text { male), } 58 \pm 10 \text { years, LVEF } 29 \% \pm 13 \% \\
\text { C: } n=23(78 \% \text { male), } 54 \pm 9 \text { years, LVEF } 30 \% \pm 11 \% \\
\text { NYHA Class } \|-I I I\end{array}$ & $\begin{array}{l}12 \text { weeks } \\
\text { Aerobic }\end{array}$ \\
\hline Nilsson et $a l^{55}$ & $\mathrm{RCT}$ & $\begin{array}{l}n=78 \text { randomised, } n=70 \text { for BNP at follow-up } \\
E: n=39(77 \% \text { male), } 69 \pm 8 \text { years, LVEF } 30 \% \pm 8 \% \\
\text { C: } n=39 \text { (79\% male), } 72 \pm 8 \text { years, LVEF } 31 \% \pm 10 \% \\
\text { NYHA Class II-III }\end{array}$ & $\begin{array}{l}4 \text { months } \\
\text { Aerobic }\end{array}$ \\
\hline Norman et $a l^{62}$ & RCT & $\begin{array}{l}n=42 \text { randomised, } n=39 \text { analysed for BNP } \\
E: n=20(55 \% \text { male), } 56 \pm 3 \text { years, LVEF } 34 \% \pm 1 \% \\
\text { C: } n=20 \text { ( } 60 \% \text { male), } 63 \pm 3 \text { years, LVEF } 32 \% \pm 1 \% \\
\text { NYHA Class II-IV }\end{array}$ & $\begin{array}{l}24 \text { weeks } \\
\text { Combined }\end{array}$ \\
\hline Palau et $a l^{42}$ & $\mathrm{RCT}$ & $\begin{array}{l}n=27 \text { randomised, } n=26 \text { analysed } \\
E: ~ n=14\left(50 \% \text { male), } 68(60-76) \text { years, LVEF } 69 \%(63-77)^{\star}\right. \\
\text { C: } n=12(50 \% \text { male), } 74 \text { (73-77) years, LVEF } 76 \%(68-83) \\
\text { NYHA Class II-IV }\end{array}$ & $\begin{array}{l}12 \text { weeks } \\
\text { IMT }\end{array}$ \\
\hline Parrinello et $a l^{63}$ & $\mathrm{RCT}$ & $\begin{array}{l}n=22 \text { randomised and analysed } \\
E: n=11(73 \% \text { male), } 62 \pm 5 \text { years, LVEF } 39 \% \pm 4 \% \\
\text { C: } n=11 \text { ( } 64 \% \text { male), } 63 \pm 5 \text { years, LVEF } 39 \% \pm 4 \% \\
\text { NYHA Class II-III }\end{array}$ & $\begin{array}{l}10 \text { weeks } \\
\text { Aerobic }\end{array}$ \\
\hline Passino et $a l^{33}$ & $\mathrm{RCT}$ & $\begin{array}{l}n=97 \text { randomised, } n=90 \text { analysed } \\
E: n=71(87 \% \text { male), } 61 \pm 2 \text { years, LVEF } 35 \% \pm 1 \% \\
\text { C: } n=19(74 \% \text { male), } 63 \pm 2 \text { years, LVEF } 36 \% \pm 2 \% \\
\text { NYHA Class I-III }\end{array}$ & $\begin{array}{l}9 \text { months } \\
\text { Aerobic }\end{array}$ \\
\hline $\begin{array}{l}\text { Sandri et } a l^{51} \\
\text { LEICA Study }\end{array}$ & $\mathrm{RCT}$ & $\begin{array}{l}n=60 \text { randomised and analysed } \\
\text { E1: } n=15 \text { (80\% male), 50 } 5 \text { years, LVEF } 27 \% \pm 1 \% \\
\text { C1: } n=15 \text { (87\% male), 49 } 45 \text { years, LVEF } 28 \% \pm 1 \% \\
\text { E2: } n=15(80 \% \text { male), } 72 \pm 4 \text { years, LVEF } 29 \% \pm 2 \% \\
\text { C2: } n=15 \text { (80\% male), } 72 \pm 3 \text { years, LVEF } 28 \% \pm 2 \% \\
\text { NYHA Class II-III }\end{array}$ & $\begin{array}{l}4 \text { weeks } \\
\text { Aerobic }\end{array}$ \\
\hline Maria Sarullo et $\left.a\right|^{52}$ & RCT & $\begin{array}{l}n=60 \text { randomised and analysed } \\
E: n=30 \text { (77\% male), } 53 \pm 6 \text { years, LVEF } 29 \% \pm 5 \% \\
\text { C: } n=30 \text { (74\% male), } 53 \pm 5 \text { years, LVEF } 29 \% \pm 4 \% \\
\text { NYHA Class II-III }\end{array}$ & $\begin{array}{l}12 \text { weeks } \\
\text { Aerobic }\end{array}$ \\
\hline Stevens et $\left.a\right|^{64}$ & RCT & $\begin{array}{l}\mathrm{n}=28 \text { randomised, } \mathrm{n}=22 \text { analysed } \\
\mathrm{E}: \mathrm{n}=15(67 \% \text { male), } 67 \pm 3 \text { years, LVEF } 39 \% \pm 3 \% \\
\text { C: } \mathrm{n}=7 \text { ( } 86 \% \text { male), } 64 \pm 6 \text { years, LVEF } 35 \% \pm 2 \% \\
\text { NYHA Class I-III }\end{array}$ & $\begin{array}{l}12 \text { weeks } \\
\text { Combined }\end{array}$ \\
\hline $\begin{array}{l}\text { Trippel et al } \\
\text { Ex-DHF pilot study post hoc } \\
\text { analysis }\end{array}$ & RCT & $\begin{array}{l}n=67 \text { randomised, } n=62 \text { analysed for biomarkers } \\
E: ~ n=44(45 \% \text { male), } 64 \pm 8 \text { years, LVEF } 68 \% \pm 7 \% \\
\text { C: } n=20 \text { ( } 40 \% \text { male), } 65 \pm 6 \text { years, LVEF } 67 \% \pm 7 \% \\
\text { NYHA Class II-III }\end{array}$ & $\begin{array}{l}12 \text { weeks } \\
\text { Combined }\end{array}$ \\
\hline
\end{tabular}




\begin{tabular}{|c|c|c|c|}
\hline Study & Design & Participant characteristics & Intervention \\
\hline Wisløff et $a l^{47}$ & RCT & $\begin{array}{l}n=27 \text { randomised, } n=26 \text { analysed } \\
\text { E1: } n=9(78 \% \text { male), } 77 \pm 9 \text { years, LVEF } 28 \% \pm 7 \% \\
\text { E2: } n=9(78 \% \text { male), } 74 \pm 12 \text { years, LVEF } 33 \% \pm 5 \% \\
\text { C: } n=9(67 \% \text { male), } 76 \pm 13 \text { years, LVEF } 26 \% \pm 8 \%\end{array}$ & $\begin{array}{l}12 \text { weeks } \\
\text { Aerobic (E1: AIT, E2: MCT) }\end{array}$ \\
\hline Yamamoto et $a l^{36}$ & $\begin{array}{l}\text { Non-RCT } \\
\text { Cohort with control } \\
\text { group }\end{array}$ & $\begin{array}{l}n=18 \text { enrolled and analysed } \\
E: n=10\left(90 \% \text { male), } 68(64-70) \text { years, LVEF } 40 \%(37-43)^{*}\right. \\
\text { C: } n=8 \text { (100\% male), } 70(66-73) \text { years, LVEF } 37 \%(35-38) \\
\text { NYHA Class II-III }\end{array}$ & $\begin{array}{l}6 \text { months } \\
\text { Aerobic }\end{array}$ \\
\hline Yeh et $a l^{69}$ & RCT & $\begin{array}{l}n=30 \text { randomised and analysed } \\
E: n=15(67 \% \text { male), } 66 \pm 12 \text { years, LVEF } 24 \% \pm 7 \% \\
\text { C: } n=15 \text { ( } 60 \% \text { male), } 61 \pm 14 \text { years, LVEF } 22 \% \pm 8 \% \\
\text { NYHA Class I-IV }\end{array}$ & $\begin{array}{l}12 \text { weeks } \\
\text { Tai Chi }\end{array}$ \\
\hline Yeh et $a l^{70}$ & RCT & $\begin{array}{l}n=100 \text { randomised and analysed } \\
E: n=50 \text { (56\% male), } 68 \pm 12 \text { years, LVEF } 28 \% \pm 8 \% \\
\text { C: } n=50 \text { (72\% male), } 67 \pm 12 \text { years, LVEF } 30 \% \pm 7 \% \\
\text { NYHA Class I-III }\end{array}$ & $\begin{array}{l}12 \text { weeks } \\
\text { Tai Chi }\end{array}$ \\
\hline
\end{tabular}

${ }^{*}$ Median (IQR).

†Randomised between three exercise groups, but control group not randomised.

†Excludes diet and diet and exercise groups.

AIT, aerobic interval training; BNP, brain natriuretic peptide; C, control; CAE, continuous aerobic training; DHF, diastolic heart failure; E, exercise; FES, functional electrical stimulation; IAE, aerobic interval training; IMT, inspiratory muscle training; LVEF, left ventricular ejection fraction; MCT, moderate continuous training; NYHA, New York Heart Association; RCT, randomised controlled trial.

demonstrated a statistically significant improvement in BNP (pmol/L) in favour of exercise; MD -17.17 (95\% CI -29.56 to -4.78 ), $\mathrm{p}=0.007$ (figure 5 ). Sensitivity analyses using the leave-one-out approach revealed that the study by Gary et $a b^{\tilde{p}}$ impacted the size of the result, with an increase in MD and statistical significance with removal of this study (figure 6).
An additional five 2236394065 studies using conventional training (table 2) reported on BNP concentrations, but were not pooled due to differences in data reporting. Two ${ }^{22} 36$ studies reported data as median (IQR), two ${ }^{39} 40$ studies were in participants with HFpEF and one ${ }^{65}$ study did not provide post-data but noted no change. Of the five studies, two ${ }^{22} 36$ reported decreases post-training in

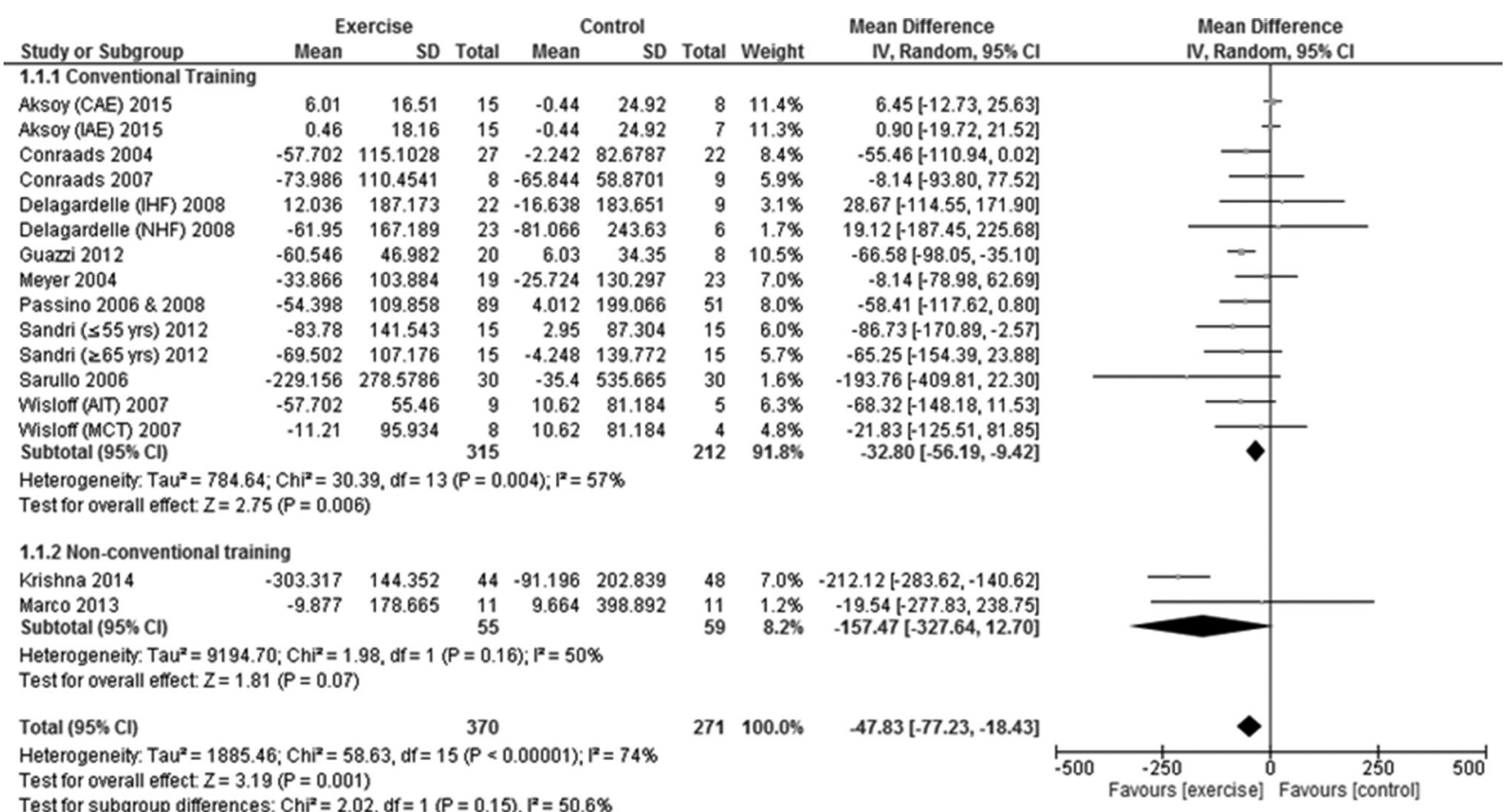

Figure 2 Change (MD) in NT-proBNP (pmol/L) exercise versus control. For conversion to $\mathrm{pg} / \mathrm{mL}=\mathrm{pmol} / \mathrm{L}$ divided by 0.118 . AIT, aerobic interval training; CAE, continuous aerobic training; IAE, aerobic interval training; IHF, ischaemic heart failure; MCT, moderate continuous training; MD, mean difference; NHF, non-ischaemic heart failure; NT-proBNP, amino (N) portion of BNP. 


\section{NT-proBNP (Conventional)}

\begin{tabular}{|c|c|c|c|c|c|}
\hline \multirow[t]{2}{*}{ Study name } & \multirow[b]{2}{*}{ Point } & \multicolumn{3}{|c|}{ Statistics with study removed } & \\
\hline & & $\begin{array}{l}\text { Standard } \\
\text { error }\end{array}$ & $\begin{array}{l}\text { Lower } \\
\text { limit }\end{array}$ & $\begin{array}{c}\text { Upper } \\
\text { limit }\end{array}$ & \\
\hline Aksoy (CAE) 2015 & -40.328 & 12.957 & -65.723 & -14.932 & \\
\hline Aksoy (IAE) 2015 & -39.865 & 14.087 & -67.478 & -12.255 & \\
\hline Corragds 2004 & -30.605 & 12.538 & -55.175 & -6.035 & \\
\hline Corragds 2007 & -34.571 & 12.587 & -59.202 & -9.939 & \\
\hline Delagar delle (IHF) 2008 & -34.489 & 12.238 & -58.472 & -10.508 & \\
\hline Delogar delle (NHF) 2008 & -33.677 & 12.197 & -57.582 & -9.771 & \\
\hline Guazzi 2012 & -22.835 & 10.702 & -43.810 & -1.881 & \\
\hline Meyes 2004 & -35.059 & 12.718 & -59.985 & -10.133 & \\
\hline Pass ino $2008 / 2008$ & -30.453 & 12.481 & -54.875 & -6.030 & \\
\hline Sendri (<55y rs) & -29.441 & 11.994 & -52949 & -5.933 & \\
\hline Sendri (> e5y rs) & -31.158 & 12.297 & -55.259 & -7.058 & \\
\hline Sarullo 2008 & -30.701 & 11.683 & -53.599 & -7.802 & \\
\hline Wisloff (AIT) 2007 & -30.580 & 12.270 & -54.629 & -6.532 & \\
\hline \multirow[t]{2}{*}{ Wisloff (MCT) 2007} & -33.588 & 12.450 & -57.989 & -9.187 & \\
\hline & -32803 & 11.932 & -58.189 & -9.418 & \\
\hline
\end{tabular}

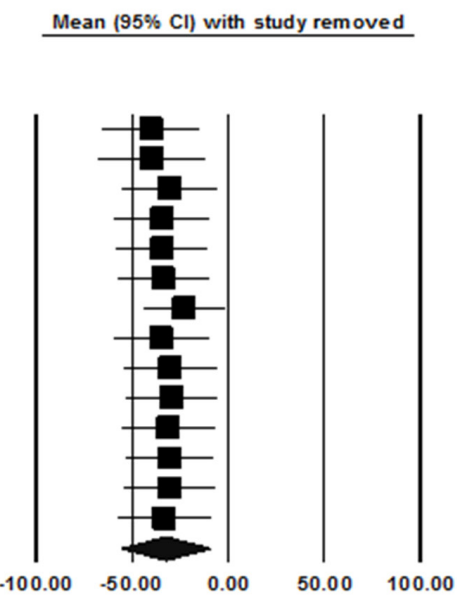

Favours Intervention Favours Control

Figure 3 Sensitivity analysis NT-proBNP (conventional training) with study removed. AIT, aerobic interval training; CAE, continuous aerobic training; IAE, aerobic interval training; IHF, ischaemic heart failure; $\mathrm{MCT}$, moderate continuous training; NHF, non-ischaemic heart failure; NT-proBNP, amino (N) portion of BNP.

exercise participants with no change in controls. The two $^{39} 40$ studies with HFpEF patents failed to find any change.
Non-conventional training

Pooled data from 4 studies $^{66-69}$ (5 intervention groups, 86 exercise participants and 59 controls) failed to

\begin{tabular}{|c|c|c|c|c|}
\hline Study & Design & Intervention & Analysed E/C & Result \\
\hline \multicolumn{5}{|l|}{ NT-proBNP } \\
\hline \multicolumn{5}{|l|}{ Conventional training } \\
\hline Ahmad et $a l^{19}$ & RCT & Aerobic & $477 / 451$ & $\leftrightarrow$ between groups \\
\hline Antonicelli et al ${ }^{44}$ & $\mathrm{RCT}$ & Aerobic & $170 / 173$ & $\downarrow$ in $\mathrm{E}$ and significantly different to $\mathrm{C}$ \\
\hline Van Berendoncks et $a /^{34}$ & Controlled & Aerobic and combined & $46 / 34$ & $\downarrow$ in $\mathrm{E}$, but $\leftrightarrow$ for $\Delta$ between $\mathrm{E}$ and $\mathrm{C}$ \\
\hline Edelmann et $a l^{30}$ & RCT & Combined & $44 / 20$ & $\leftrightarrow$ in $\mathrm{E}$ or $\mathrm{C}$ \\
\hline Eleuteri et $a l^{54}$ & RCT & Aerobic & $11 / 10$ & $\leftrightarrow$ in $\mathrm{E}$ or $\mathrm{C}$ \\
\hline Nilsson et a $\left.\right|^{55}$ & RCT & Aerobic & $37 / 33$ & $\leftrightarrow$ in $\mathrm{E}$ or $\mathrm{C}$ or between $\mathrm{E}$ and $\mathrm{C}$ \\
\hline \multicolumn{5}{|l|}{ Non-conventional } \\
\hline Palau et $a 4^{42}$ & RCT & IMT & $14 / 12$ & $\leftrightarrow$ in $\mathrm{E}$ or $\mathrm{C}$ or between $\mathrm{E}$ and $\mathrm{C}$ \\
\hline \multicolumn{5}{|l|}{ BNP } \\
\hline \multicolumn{5}{|l|}{ Conventional training } \\
\hline Billebeau et $a l^{22}$ & Controlled & Aerobic & $107 / 24$ & $\downarrow$ in $E, \leftrightarrow$ in $C$ \\
\hline Brubaker et a ${ }^{65}$ & RCT & Aerobic & $23 / 21$ & $\leftrightarrow$ between $\mathrm{E}$ and $\mathrm{C}$ \\
\hline Kitzman et $a l^{40}$ & RCT & Aerobic & $26 / 27$ & $\leftrightarrow$ between $\mathrm{E}$ and $\mathrm{C}$ \\
\hline Kitzman et $a l^{39}$ & RCT & Aerobic & $26 / 25$ & $\leftrightarrow$ in $\mathrm{E}$ or $\mathrm{C}$ \\
\hline Yamamoto et $\left.a\right|^{36}$ & Controlled & Aerobic & $10 / 8$ & $\downarrow$ in $E, \leftrightarrow$ in $C$ \\
\hline \multicolumn{5}{|l|}{ Non-conventional } \\
\hline Karavidas et al ${ }^{41}$ & RCT & FES & $15 / 15$ & $\leftrightarrow$ for $\Delta$ between $\mathrm{E}$ and $\mathrm{C}$ \\
\hline Yeh et $a l^{70}$ & RCT & Tai Chi & $50 / 50$ & $\leftrightarrow$ for $\Delta$ between $\mathrm{E}$ and $\mathrm{C}$ \\
\hline
\end{tabular}

$\downarrow$ statistically significant, $\leftrightarrow$ no statistically significant change.

BNP, brain natriuretic peptide; C, control; E, exercise; FES, functional electrical stimulation; IMT, inspiratory muscle training; NT-

proBNP, amino (N) portion of BNP; RCT, randomised controlled trial. 


\section{NT-proBNP (Non Conventional)}

\begin{tabular}{|c|c|c|c|c|c|}
\hline \multirow[t]{2}{*}{ Study name } & \multirow[b]{2}{*}{ Point } & \multicolumn{3}{|c|}{ Statistics with study removed } & \multirow[b]{2}{*}{ p-Value } \\
\hline & & $\begin{array}{l}\text { Standard } \\
\text { error }\end{array}$ & $\begin{array}{c}\text { Lower } \\
\text { limit }\end{array}$ & $\begin{array}{c}\text { Upper } \\
\text { limit }\end{array}$ & \\
\hline Krishna 2014 & -19.540 & 131.784 & -277.831 & 238.751 & 0.882 \\
\hline \multirow[t]{2}{*}{ Marco 2013} & -212.200 & 36.479 & -283.698 & -140.702 & 0.000 \\
\hline & -157.488 & 86.876 & -327.761 & 12.786 & 0.070 \\
\hline
\end{tabular}

Mean $(95 \% \mathrm{Cl})$ with study removed

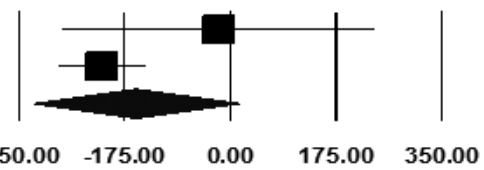

Fav ours Intervention Fav ours Control

Figure 4 Sensitivity analysis NT-proBNP (non-conventional training) with study removed. NT-proBNP, amino (N) portion of BNP.

demonstrate a statistically significant improvement in $\mathrm{BNP}$ (pmol/L) exercise versus control; MD -9.92 (95\% CI -28.03 to -8.20 ), $\mathrm{p}=0.28$ (figure 5 ). Sensitivity analysis indicated that the study by Kawauchi et al ${ }^{68}$ affected the magnitude of the result (figure 7). Sensitivity analyses conducted for different correlation coefficients for SD imputation did not result in any significant variance in overall results. Two ${ }^{41} 70$ additional studies, using non-conventional training, were not pooled. One ${ }^{70}$ reported data as median (IQR), and one ${ }^{41}$ was in patients with $\mathrm{HFpEF}$, and both failed to demonstrate any significant change (table 2).

\section{Cardiac troponin}

Only a substudy of the HF ACTION trial reported on the effect of exercise training on cTnT levels compared with control participants, with no decreases in detectable levels of cTnT found in a cohort of participants from the trial. ${ }^{53}$

\section{Galectin-3}

Two studies compared Gal-3 in exercising and control participants. However, differences in data reporting did not allow for data pooling. Billebeau et $a t^{22}$ observed a statistically significant $(\mathrm{p}<0.001)$ median decrease of $6.3 \%$ in the exercise group $(n=107)$ with no change in control patients. While Fernandes-Silva et $a t^{23}$ reported no statistically significant difference in the mean change between exercise and control groups $(p=0.69)$.

\section{Soluble ST2}

One study reported predata and postdata in regard to the effect of exercise training on sST2 levels. A statistically

\begin{tabular}{|c|c|c|c|c|c|c|c|c|c|c|c|}
\hline \multirow[b]{2}{*}{ Study or Subgroup } & \multicolumn{3}{|c|}{ Exercise } & \multicolumn{3}{|c|}{ Control } & \multicolumn{2}{|r|}{ Mean Difference } & \multirow{2}{*}{\multicolumn{3}{|c|}{$\begin{array}{c}\text { Mean Difference } \\
\text { IV, Random, } 95 \% \mathrm{CI}\end{array}$}} \\
\hline & Mean & SD & Total & Mean & SD & Total & Weight & IV, Random, 95\% Cl & & & \\
\hline \multicolumn{12}{|c|}{ 2.1.1 Conventional Training } \\
\hline Butterfield 2008 & 5.702 & 39.714 & 11 & 31.26 & 74.552 & 6 & $2.1 \%$ & $-25.56[-89.66,38.55]$ & & & \\
\hline Fu (AIT) 2013 & -55.488 & 37.192 & 14 & -0.578 & 75.809 & 7 & $2.4 \%$ & $-54.91[-114.35,4.53]$ & & & \\
\hline Gary 2011 & 35.72 & 46.124 & 12 & 0.75 & 30.143 & 12 & $6.5 \%$ & $34.97[3.79,66.15]$ & & & \\
\hline Jonsdottir 2006 & -0.434 & 48.892 & 21 & 0.665 & 40.794 & 22 & $7.7 \%$ & $-1.10[-28.08,25.88]$ & & & \\
\hline Kobayashi 2003 & -4.046 & 94.541 & 14 & 0 & 124.476 & 14 & $1.4 \%$ & $-4.05[-85.92,77.83]$ & & & \\
\hline Malfatto 2009 & -36.992 & 31.408 & 27 & -0.867 & 35.157 & 27 & $11.3 \%$ & $-36.13[-53.91,-18.34]$ & & & \\
\hline Norman 2012 & -3.699 & 30.579 & 20 & 7.716 & 57.926 & 19 & $7.0 \%$ & $-11.41[-40.71,17.88]$ & & & \\
\hline Parrinello 2009 & -11.416 & 12.561 & 11 & 8.497 & 13.96 & 11 & $14.3 \%$ & $-19.91[-31.01,-8.82]$ & - & & \\
\hline Passino 2006 \& 2008 & -18.496 & 39.015 & 89 & 1.445 & 43.928 & 51 & $12.8 \%$ & $-19.94[-34.47,-5.41]$ & & & \\
\hline $\begin{array}{l}\text { Stevens } 2015 \\
\text { Subtotal }(95 \% \mathrm{Cl})\end{array}$ & -11.849 & 108.051 & $\begin{array}{r}15 \\
268\end{array}$ & 17.918 & 106.99 & $\begin{array}{r}7 \\
192\end{array}$ & $\begin{array}{r}1.0 \% \\
70.2 \%\end{array}$ & $\begin{array}{r}-29.77[-126.06,66.52] \\
-17.17[-29.56,-4.78]\end{array}$ & & & \\
\hline \multicolumn{12}{|c|}{$\begin{array}{l}\text { Heterogeneity: } \text { Tau }^{2}=170.39 ; \mathrm{Ch}^{2}=21.36, \mathrm{df}=11(P=0.03) ; \mathrm{I}^{2}=48 \% \\
\text { Test for overall effect: } Z=2.72(P=0.007)\end{array}$} \\
\hline \multicolumn{12}{|c|}{ 2.1.2 Non-conventional Training } \\
\hline Kato 2017 & -1.3 & 51.876 & 25 & 12.572 & 63.841 & 25 & $6.2 \%$ & $-13.87[-46.12,18.37]$ & & & \\
\hline Kawauchi (LIPRT) 2017 & -6.358 & 65.025 & 13 & -7.225 & 18.496 & 5 & $4.8 \%$ & $0.87[-38.02,39.75]$ & & & \\
\hline Kawauchi (MIPRT) 2017 & 18.496 & 71.094 & 13 & -7.225 & 18.496 & 4 & $4.2 \%$ & $25.72[-16.96,68.41]$ & & & \\
\hline $\begin{array}{l}\text { Yeh } 2004 \\
\text { Subtotal }(95 \% \mathrm{Cl})\end{array}$ & -13.872 & 48.0538 & $\begin{array}{l}15 \\
86\end{array}$ & 26.01 & 48.0538 & $\begin{array}{l}15 \\
59\end{array}$ & $\begin{array}{r}5.7 \% \\
29.8 \%\end{array}$ & $\begin{array}{r}-39.88[-74.27,-5.49] \\
-9.92[-28.03,8.20]\end{array}$ & & & \\
\hline \multicolumn{12}{|c|}{$\begin{array}{l}\text { Heterogeneity: } \text { Tau }^{2}=138.61 ; \mathrm{Chi}^{2}=5.94, \mathrm{df}=4(P=0.20) ; \mathrm{I}^{2}=33 \% \\
\text { Test for overall effect: } Z=1.07(P=0.28)\end{array}$} \\
\hline Total $(95 \% \mathrm{Cl})$ & & & 354 & & & 251 & $100.0 \%$ & $-15.02[-25.06,-4.99]$ & & & \\
\hline \multicolumn{9}{|c|}{$\begin{array}{l}\text { Heterogeneity: } \operatorname{Tau}^{2}=150.67 ; \mathrm{Chi}^{2}=28.35, \mathrm{df}=16(P=0.03) ; \mathrm{I}^{2}=44 \% \\
\text { Test for overall effect } Z=2.93(P=0.003) \\
\text { Test for subaroup differences: } \mathrm{Ch}^{2}=0.42, \mathrm{df}=1(\mathrm{P}=0.52), \mathrm{I}^{\mathrm{z}}=0 \%\end{array}$} & $\begin{array}{c}-100 \\
\text { Favours le }\end{array}$ & Favo & $\begin{array}{l}100 \\
\text { [control] }\end{array}$ \\
\hline
\end{tabular}

Figure 5 Change (MD) in BNP (pmol/L) exercise versus control. For conversion to $\mathrm{pg} / \mathrm{mL}=\mathrm{pmol} / \mathrm{L}$ divided by 0.289 . AIT, aerobic interval training; BNP, brain natriuretic peptide; LIPRT, low-intensity inspiratory training and peripheral resistance training; $\mathrm{MCT}$, moderate continuous training; MD, mean difference MIPRT, moderate-intensity inspiratory and peripheral resistance training. 


\section{BNP (Conventional)}

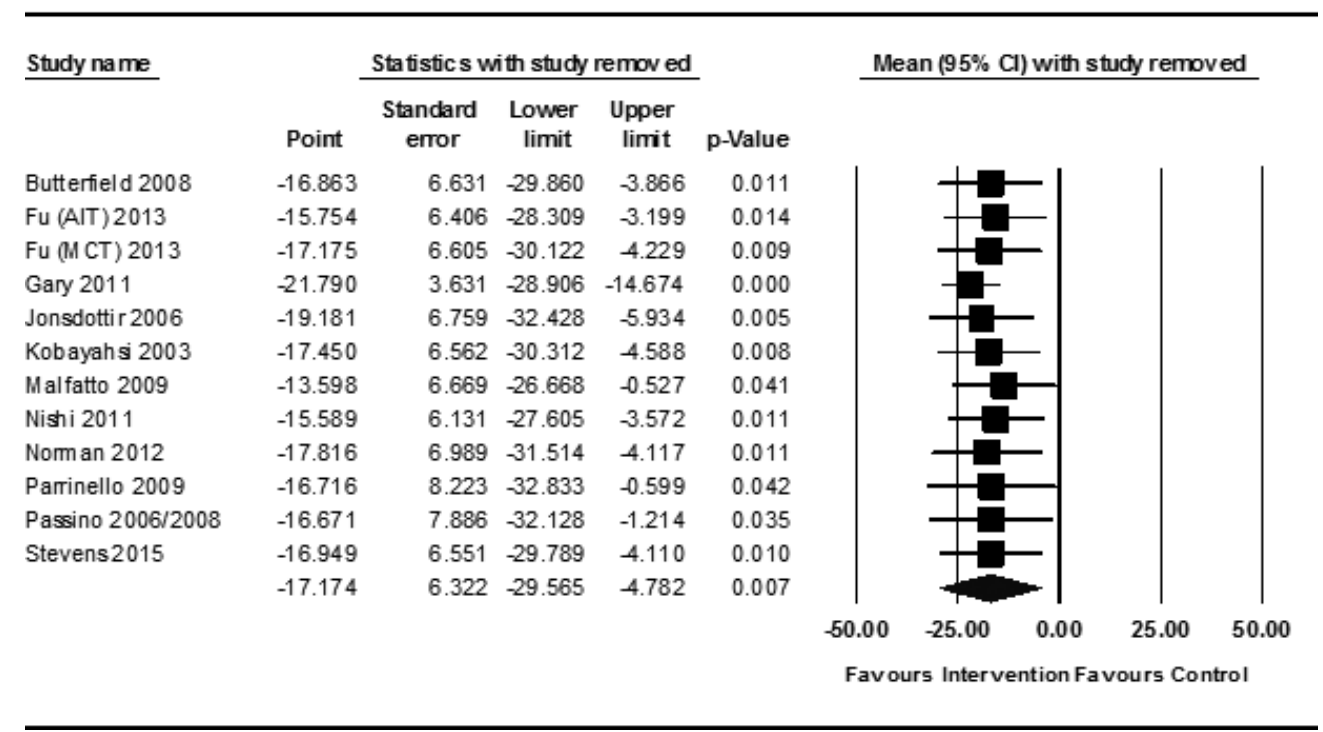

Figure 6 Sensitivity analysis BNP (conventional training) with study removed. AIT, aerobic interval training; BNP, brain natriuretic peptide; MCT, moderate continuous training.

significant $(p=0.035)$ median decrease of $7.4 \%$ was observed post-training $(\mathrm{n}=97)$ by Billebeau et $a l^{22}$ with no change in controls.

\section{MR-proANP}

Two studies reported on postintervention MR-proANP concentrations. Billebeau et $a t^{22}$ observed a statistically significant $(\mathrm{p}<0.001)$ median decrease of $16 \%$ posttraining $(\mathrm{n}=105)$, with no changes in control participants. In contrast, the post hoc analysis of the Ex-DHF pilot trial by Trippel et $a l^{\hat{1}}$ noted no significant treatment effect in patients with HFpEF.
Mid-regional adrenomedullin

Two studies reported on postintervention MR-proADM concentrations. Billebeau et $a l^{22}$ observed a statistically significant $(p=0.001) 6.4 \%$ median decrease in MR-proADM ( $\mathrm{n}=103)$, with no changes in control participants. In contrast, Trippel $e t a l^{31}$ noted no significant treatment effect in patients with HFpEF.

Copeptin

One study by Trippel $e t a \ell^{31}$ reported on CT-proAVP levels and failed to find any statistically significant change posttraining or compared with the control group in patients with HFpEF.

\section{BNP (Non Conventional)}

\begin{tabular}{|c|c|c|c|c|c|}
\hline \multirow[t]{2}{*}{ Study name } & \multirow[b]{2}{*}{ Point } & \multicolumn{3}{|c|}{ Statistics with study removed } & \multirow[b]{2}{*}{ p-Value } \\
\hline & & $\begin{array}{c}\text { Standard } \\
\text { error }\end{array}$ & $\begin{array}{l}\text { Lower } \\
\text { limit }\end{array}$ & $\begin{array}{l}\text { Upper } \\
\text { limit }\end{array}$ & \\
\hline Karav idss 2008 & -8.912 & 13.198 & -34.771 & 16.946 & 0.499 \\
\hline Kato 2017 & -8.265 & 12.137 & -32.053 & 15.523 & 0.498 \\
\hline Kawauchi (LIPRT) 2017 & -11.713 & 11.211 & -33.687 & 10.260 & 0.298 \\
\hline Kgwauchi (MIPRT) 2017 & -15.284 & 7.823 & -30.617 & 0.049 & 0.051 \\
\hline \multirow[t]{2}{*}{ Yeh 2004} & -4.341 & 8.111 & -20.238 & 11.558 & 0.592 \\
\hline & -9.918 & 9.241 & -28.028 & 8.196 & 0.283 \\
\hline
\end{tabular}

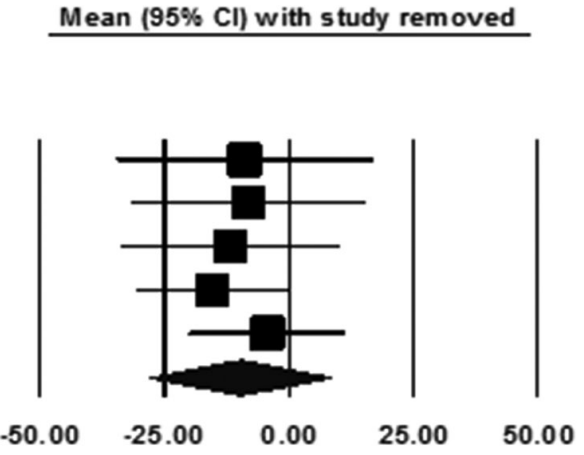

Favours Intervention Favours Control

Figure 7 Sensitivity analysis BNP (non-conventional training) with study removed. BNP, brain natriuretic peptide; LIPRT, lowintensity inspiratory training and peripheral resistance training; MIPRT, moderate-intensity inspiratory and peripheral resistance training. 


\section{Study quality and reporting}

A median TESTEX score of 8.5 out of 15 was obtained (range 6-12) (online supplementary table S6). Details of randomisation procedures, activity monitoring of control groups, adjustment of relative exercise intensity and provision of adequate details to calculate exercise energy expenditure were frequently lacking.

\section{Heterogeneity and publication bias}

Meta-analyses indicated a moderate level of heterogeneity. Visual inspection of the funnel plot showed slight asymmetry (online supplementary figures $1 \mathrm{~A}, \mathrm{~B}$ ).

\section{DISCUSSION}

This systematic review and meta-analysis compiled evidence from a large volume of studies assessing the effect of exercise therapy on established and a selected number of emerging biomarkers in patients with HF. Different to previous analyses, both conventional and non-conventional modes of training were examined. When analysed separately, conventional training demonstrated a statistically significant improvement in NT-proBNP and BNP, while pooled analyses of non-conventional training failed to demonstrate any significance. While BNP and NT-proBNP are raised across the HF spectrum, as levels may be lower in HFpEF, and in some instances close to normal, we excluded studies from pooled analyses that only included patients with HFpEF. However, it is highly likely that a number of other studies included in the analyses with mean ejection fractions $>40 \%$ would have also included patients with HFpEF, and it is possible that this could be reflected in the variability of the results.

The favourable result demonstrated in pooled analyses of conventional training is consistent with previous reviews $^{24} 71$ and a 2011 IPD meta-analysis. ${ }^{25}$ However, in contrast to our pooled results, of studies unable to be pooled, only two of seven studies for BNP, and two of the seven studies for NT-proBNP, indicated any significant change post-training or compared with controls. Furthermore, one of these studies was a sub-analysis of a large cohort from the HF ACTION trial, which found that levels of plasma NT-proBNP did not significantly improve after 3 months of AT, ${ }^{53}$ clearly contrasting with our result and previous analyses. ${ }^{2425} 71$ However, adherence and participant crossover issues may have confounded the results of the HF ACTION trial. It is also possible that a longer intervention duration may have resulted in significant changes, as seen after 9 months by Passino et $a l^{32}$ although Sandri et a $\tilde{l}^{51}$ demonstrated significant decreases after only 4 weeks of endurance training.

\section{Emerging biomarkers}

While BNP/NT-proBNP remains the gold standard HF biomarkers, with proven prognostic value, there are limitations. Age, gender, arrhythmias, obesity, renal function and comorbidities ${ }^{10} 12$ may all affect concentrations; hence, biomarkers less affected by these issues can provide valuable information. Furthermore, as biomarkers of myocardial stretch, BNP/NT-proBNP is only reflective of one pathophysiological pathway involved in HF; hence, biomarkers reflecting other pathways may provide new and valuable information and complement BNP/NT-proBNP. Both Gal-3 and sST2 have been studied as emerging biomarkers in HF, and now have a Class IIB recommendation for risk stratification by the American College of Cardiology/American Heart Association (ACC/AHA) (2013) guideline for HF management. ${ }^{7}$ Gal-3, a $\beta$-galactoside-binding lectin, plays a dominant role in inflammation, fibrosis and cardiac remodelling. ${ }^{10}{ }^{12}$ sST2, a member of the interleukin (IL)-1 receptor family and defined as a ligand for IL-33, is considered a cardiovascular stress protein, associated with fibrosis, cardiac and vascular remodelling and inflammation. ${ }^{72}$ Initial evidence also indicates that other novel biomarkers, such as CT-proAVP ${ }^{2073} 74$ and MR-proADM, ${ }^{75}$ both biomarkers of neurohormonal activation, also have prognostic value in HF.

Current evidence does not allow for any conclusion as to the effect of exercise training on emerging biomarkers. However, the recent studies of Fernandes-Silva $e t a l^{23}$ and Billebeau et $a l^{22}$ provide an interesting and perhaps promising platform on which future research can expand. Billebeau $e t ~ a l,{ }^{22}$ in a non-randomised trial, observed a significant decrease in BNP, MR-proANP, MR-proADM, Gal-3 and sST2 in exercise training participants with no change in controls. Analysis according to change in $\mathrm{VO}_{\text {2peak }}$ demonstrated that patients with an increase in $\mathrm{VO}_{2 \text { peak }} \geq 14.5 \%$ (based on the median increase) experienced a significant decrease in Gal-3, sST2, MR-proADM and MR-proANP compared with no significant biomarker change in participants with change in $\mathrm{VO}_{2 \text { peak }}<14.5 \%$. $^{22}$ Furthermore, given that BNP improved regardless of the change in $\mathrm{VO}_{2 \text { peak }}$, they concluded that the addition of the newer biomarkers improved the clinical follow-up of rehabilitation. ${ }^{22}$ Overall, their results demonstrated that exercise training improves neurohormonal, inflammatory and fibrotic processes. ${ }^{22}$ Fernandes-Silva $e t a l^{23}$ observed no significant difference between exercise and control patients for change in Gal-3 or the proinflammatory markers (IL-6 and tumour necrosis factor- $\alpha$ ); however, $\mathrm{VO}_{2 \text { peak }}$ significantly improved in participants with low baseline Gal-3 levels, compared with patients with high levels, with similar findings for the proinflammatory markers. These results suggesting biomarkers may predict a patient's response to training. ${ }^{23}$ Interestingly, in a substudy of the HF ACTION trial, higher baseline ST2 levels were associated with a greater improvement in $\mathrm{VO}_{\text {2peak }}$ at 3 months. ${ }^{76}$

\section{Exercise capacity}

Reduced exercise capacity is a major hallmark of HF, and NT-proBNP is a strong predictor of $\mathrm{VO}_{2 \text { peak }}{ }^{77}$ Changes in BNP and NT-proBNP have been correlated with changes in $\mathrm{VO}_{2 \text { peak }}$ and suggested therefore as a possible surrogate for evaluating training responses. ${ }^{25}$ Only a minimal number of studies included in the review 
reported associations between change in peak $\mathrm{VO}_{2 \text { peak }}$ and biomarkers. Ahmad et $a \bar{l}^{53}$ did however observe that in patients in whom NT-proBNP levels decreased, there was an increase in $\mathrm{VO}_{\text {2peak }}$, despite finding no significant change in NT-proBNP. While Passino $e t a l^{32}$ observed that changes in $\mathrm{VO}_{2 \text { peak }}$ correlated significantly with decreases in NT-proBNP and BNP. Recently, Billebeau et al found that of all the biomarkers they tested, for predicting change in exercise capacity, MR-proADM best correlated with $\mathrm{VO}_{2 \text { peak }} \cdot{ }^{22}$ Given that adrenomedullin originates not only from the heart but also from multiple organs, tissues and blood vessels ${ }^{78}$ and that the mechanisms associated with improved exercise capacity in HF involve cardiac, vascular and skeletal muscle adaptations, ${ }^{79}$ a relationship between MR-proADM and improved exercise capacity makes sense.

\section{Phenotype}

Levels of BNP and NT-proBNP are elevated irrespective of ejection fraction; although they are generally lower in HFpEF compared with heart failure with reduced ejection fraction (HFrEF). ${ }^{80-82}$ Patients also present with elevated levels of a number of other biomarkers reflective of different pathophysiological pathways. Currently, there are limited data on the role of exercise training and biomarkers in HFpEF, and none of the HFpEF studies included in the review reported any significant changes in the biomarkers. Furthermore, it is likely that there exist different biomarker profiles for HFrEF and HFpEF. ${ }^{83} 84$ Moving forward, these different biomarker profiles may provide valuable information for treatment strategies, including exercise.

\section{Exercise prescription}

While moderate continuous training (MCT) has been the cornerstone of conventional HF training, over the past decade, the interest in high-intensity interval training (HIIT) has grown. ${ }^{85}$ Two studies included in the review that specifically incorporated HIIT and MCT groups for comparative purposes observed significant improvements in $\mathrm{BNP}^{57}$ and NT-proBNP ${ }^{47}$ from HIIT, with no significant change from MCT. However, this is in contrast to the recent results of the larger, multicentre SMARTEX HF study, which failed to demonstrate any significant difference between HIIT and MCT after 12 weeks. ${ }^{86}$ However, for comparisons, difficulty arises in regard to actual training intensities attained, and in SMARTEX, both actual HIIT and MCT intensities attained may have impacted the results, with patients training at lower and higher intensities than prescribed. ${ }^{86}$

To date, the majority of HF training studies have used conventional modes of training; however, not all patients can or are willing to participate in these activities. Women, for example, may be more likely to attend mind-body interventions, such as Tai Chi and Yoga, for cardiac rehabilitation purposes. ${ }^{878}$ Furthermore, both FES and IMT offer alternative modes of physical therapy, particularly in patients unable to participate in more conventional modalities. Individually, the included studies investigating FES and IMT failed to demonstrate any significant change in BNP or NT-proBNP compared with control groups. However, the combination of these non-conventional modes with conventional training may provide possible synergistic effects, ${ }^{89}$ as demonstrated by Caminiti et $a t^{90}$ with combined Tai Chi/endurance training and Adamopoulos et $a l^{89}$ with combined IMT/AT. Furthermore, other modes of non-conventional exercise therapy, such as weight-supported ${ }^{91}$ and robot-assisted ${ }^{92}$ exercise training, have demonstrated improvements in BNP and NT-proBNP in patients with HF and may be beneficial in some subgroups.

\section{Clinical significance and future research}

Biomarkers are used in HF clinical trials for a number of reasons, ${ }^{10}$ including establishment of inclusion criteria, outcome measures, explaining therapeutic efficacy and as a target for therapy. ${ }^{93}$ Biomarkers and biomarker panels may aid in identifying subgroups of patients with HF who may have a more favourable response to exercise therapy, distinguishing responders and non-responders ${ }^{21} 23$ in terms of specified outcomes including functional and long-term outcomes. Different biomarkers may provide further insight into the downstream molecular mechanisms associated with improvements from exercise training. ${ }^{21}$ It could be possible that different biomarker profiles respond differently to different intervention characteristics, such as intensity, perhaps allowing further tailoring of the exercise to the individual. Furthermore, biomarkers, with their prognostic utility, may provide useful postintervention information, indicating improvements when other favourable outcomes may be absent. It remains premature to draw too many conclusions about the relationship between changes in emerging biomarkers and exercise training, and the utility of these biomarkers in HF is yet to be fully established, but it presents as an interesting and important area for future research.

Future research also needs to consider the clinical interpretation of changes in biomarkers given their biological variation. ${ }^{94}$ While NT-proBNP is considered to have high biological variation, the newer markers of sST2 and Gal-3 demonstrate a lower variation and therefore add value to their use. ${ }^{94}$ However, from an individual perspective in interpreting clinically meaningful changes in biomarkers, it is suggested that reference change values which indicate the percentage change necessary within an individual, reflective of a true change as opposed to biological variation, be used. ${ }^{94}$

\section{Strengths and limitations in the systematic review and meta- analysis}

To our knowledge, this is the first meta-analysis of BNP and NT-proBNP to include training studies beyond the conventional AT and RT modalities and the first review to consider exercise therapy and emerging biomarkers in HF. We aimed to provide a meta-analysis of studies 
reporting on a selected number of established and emerging biomarkers. However, as biomarker distributions can be skewed, study data may often be presented as median (IQR) or median (range), which precludes it from inclusion in meta-analyses. Valuable information may be ignored if a number of studies are excluded; therefore, on initial review and identification of a number of studies that had examined biomarkers and reported data as median or provided a descriptive result, we felt that the inclusion of these studies would enhance the value of the review and analysis. Therefore, we included results of studies reporting data that were considered inappropriate for pooling and only provided a descriptive analysis of these studies.

Studies in which biomarkers were assessed as secondary outcomes may not have been adequately powered to detect significant differences in biomarkers. Furthermore, the studies included in the review reported a wide range of intervention durations, training frequency, session times and intensity. In regard to data pooling, we measured the difference between preintervention and postintervention means; however, in cases where exact $\mathrm{p}$ values within groups or $95 \% \mathrm{CI}$ were not available, we imputed the SD, and hence statistical analysis depended on extrapolated data. However, our imputation was conservative, and sensitivity analyses were conducted for different correlation coefficients. Abstracts and trials not reported in English were excluded and could have led to publication bias.

\section{CONCLUSION}

Pooled data of conventional training modalities indicated a favourable effect on the established HF biomarkers, NT-proBNP and BNP, contrasting with information from a number of non-pooled studies. Limited evidence exists in regard to exercise training and emerging biomarkers. Given the complex pathways involved in the onset and progression of $\mathrm{HF}$, more research is required to establish exactly how established and emerging biomarkers can be used in exercise training in this population. The use of multiple biomarkers is an area of active research in $\mathrm{HF}$, and future studies using biomarker panels may prove beneficial in guiding non-pharmacological therapy such as exercise by facilitating a more precise approach to exercise for subgroups of patients.

Contributors MJP designed the review, conducted the literature search, extracted data, undertook data analysis and wrote the manuscript. NK assisted with the preparation of the study quality assessment and review of the manuscript. NAS assisted in selecting eligible articles and reviewed and edited the manuscript. All authors approved the final manuscript.

Funding MJP is supported by an Australian Postgraduate Award Scholarship.

Disclaimer The authors take responsibility for all aspects of the reliability and freedom from bias of the data presented and their discussed interpretation.

Competing interests None declared.

Patient consent Not required.

Provenance and peer review Not commissioned; externally peer reviewed.
Data sharing statement No additional data are available.

Open access This is an open access article distributed in accordance with the Creative Commons Attribution Non Commercial (CC BY-NC 4.0) license, which permits others to distribute, remix, adapt, build upon this work non-commercially, and license their derivative works on different terms, provided the original work is properly cited and the use is non-commercial. See: http://creativecommons.org/ licenses/by-nc/4.0/

(C) Article author(s) (or their employer(s) unless otherwise stated in the text of the article) 2018. All rights reserved. No commercial use is permitted unless otherwise expressly granted.

\section{REFERENCES}

1. Taylor RS, Sagar VA, Davies EJ, et al. Exercise-based rehabilitation for heart failure. The Cochrane Library 2014.

2. Ismail $\mathrm{H}$, McFarlane JR, Nojoumian $\mathrm{AH}$, et al. Clinical outcomes and cardiovascular responses to different exercise training intensities in patients with heart failure: a systematic review and meta-analysis. JACC Heart Fail 2013;1:514-22.

3. Pearson MJ, Smart NA. Exercise therapy and autonomic function in heart failure patients: a systematic review and meta-analysis. Heart Fail Rev 2018;23:1-18.

4. Dieberg G, Ismail H, Giallauria F, et al. Clinical outcomes and cardiovascular responses to exercise training in heart failure patients with preserved ejection fraction: a systematic review and metaanalysis. J Appl Physiol 2015;119:726-33.

5. Pearson MJ, Smart NA. Effect of exercise training on endothelial function in heart failure patients: A systematic review meta-analysis. Int J Cardiol 2017;231:234-43.

6. Ponikowski P, Voors AA, Anker SD, et al. 2016 ESC Guidelines for the diagnosis and treatment of acute and chronic heart failure. Eur Heart J 2016;37:2129-200.

7. Yancy CW, Jessup M, Bozkurt B, et al. 2013 ACCF/AHA guideline for the management of heart failure: a report of the American College of Cardiology Foundation/American Heart Association Task Force on Practice Guidelines. J Am Coll Cardiol 2013;62:e147-239.

8. Senthong V, Kirsop JL, Tang WH. Clinical Phenotyping of Heart Failure with Biomarkers: Current and Future Perspectives. Curr Heart Fail Rep 2017;14:106-16.

9. Cypen J, Ahmad T, Testani JM, et al. Novel Biomarkers for the Risk Stratification of Heart Failure with Preserved Ejection Fraction. Curr Heart Fail Rep 2017;14:434-43.

10. Chow SL, Maisel AS, Anand I, et al. Role of Biomarkers for the Prevention, Assessment, and Management of Heart Failure: A Scientific Statement From the American Heart Association. Circulation 2017;135:e1054-91.

11. Yancy CW, Jessup M, Bozkurt B, et al. 2017 ACC/AHA HFSA Focused Update of the 2013 ACCF/AHA Guideline for the Management of Heart Failure. J Card Fail 2017;23:628-51.

12. Berezin AE. Up-to-date clinical approaches of biomarkers' use in heart failure. Biomedical Research and Therapy 2017;4:1344-73.

13. Braunwald E. Heart Failure. JACC Heart Fail 2013;1:1-20.

14. Aimo A, Vergaro G, Passino C, et al. Prognostic value of soluble suppression of tumorigenicity-2 in chronic heart failure: a metaanalysis. JACC Heart Fail 2017;5:280-6.

15. Miller WL, Saenger AK, Grill DE, et al. Prognostic value of serial measurements of soluble suppression of tumorigenicity 2 and galectin-3 in ambulatory patients with chronic heart failure. $J$ Card Fail 2016;22:249-55.

16. Imran TF, Shin HJ, Mathenge N, et al. Meta-Analysis of the Usefulness of Plasma Galectin-3 to Predict the Risk of Mortality in Patients With Heart Failure and in the General Population. Am J Cardiol 2017;119:57-64.

17. French B, Wang L, Ky B, et al. Prognostic Value of Galectin-3 for Adverse Outcomes in Chronic Heart Failure. J Card Fail 2016;22:256-62.

18. Pascual-Figal DA, Ordoñez-Llanos J, Tornel PL, et al. Soluble ST2 for predicting sudden cardiac death in patients with chronic heart failure and left ventricular systolic dysfunction. J Am Coll Cardiol 2009;54:2174-9.

19. Ahmad T, Fiuzat M, Neely B, et al. Biomarkers of myocardial stress and fibrosis as predictors of mode of death in patients with chronic heart failure. JACC Heart Fail 2014;2:260-8.

20. Zhang P, Wu X, Li G, et al. Prognostic role of copeptin with all-cause mortality after heart failure: a systematic review and meta-analysis. Ther Clin Risk Manag 2017;13:49-58.

21. Masterson Creber RM, Lee CS, Margulies K, et al. Identifying biomarker patterns and predictors of inflammation and myocardial stress. J Card Fail 2015;21:439-45. 
22. Billebeau G, Vodovar N, Sadoune M, et al. Effects of a cardiac rehabilitation programme on plasma cardiac biomarkers in patients with chronic heart failure. Eur J Prev Cardiol 2017;24:1127-35.

23. Fernandes-Silva MM, Guimarães GV, Rigaud VO, et al. Inflammatory biomarkers and effect of exercise on functional capacity in patients with heart failure: Insights from a randomized clinical trial. Eur J Prev Cardiol 2017;24:808-17.

24. Smart NA, Steele M. Systematic review of the effect of aerobic and resistance exercise training on systemic brain natriuretic peptide (BNP) and N-terminal BNP expression in heart failure patients. Int $J$ Cardiol 2010;140:260-5.

25. Smart NA, Meyer T, Butterfield JA, et al. Individual patient meta-analysis of exercise training effects on systemic brain natriuretic peptide expression in heart failure. Eur J Prev Cardiol 2012;19:428-35.

26. Fu R, Vandermeer BW, Shamliyan TA, et al. Handling continuous outcomes in quantitative synthesis, 2013.

27. Higgins JP, Thompson SG, Deeks JJ, et al. Measuring inconsistency in meta-analyses. BMJ 2003;327:557-60.

28. Egger M, Davey Smith G, Schneider M, et al. Bias in meta-analysis detected by a simple, graphical test. BMJ 1997;315:629-34.

29. Smart NA, Waldron M, Ismail H, et al. Validation of a new tool for the assessment of study quality and reporting in exercise training studies: TESTEX. Int J Evid Based Healthc 2015;13:9-18.

30. Edelmann F, Gelbrich G, Düngen HD, et al. Exercise training improves exercise capacity and diastolic function in patients with heart failure with preserved ejection fraction: results of the Ex-DHF (Exercise training in Diastolic Heart Failure) pilot study. J Am Coll Cardiol 2011;58:1780-91

31. Trippel TD, Holzendorf V, Halle M, et al. Ghrelin and hormona markers under exercise training in patients with heart failure with preserved ejection fraction: results from the Ex-DHF pilot study. ESC Heart Fail 2017:4:56-65.

32. Passino C, Severino S, Poletti R, et al. Aerobic training decreases B-type natriuretic peptide expression and adrenergic activation in patients with heart failure. J Am Coll Cardiol 2006;47:1835-9.

33. Passino C, Del Ry S, Severino S, et al. C-type natriuretic peptide expression in patients with chronic heart failure: effects of aerobic training. Eur J Cardiovasc Prev Rehabil 2008;15:168-72.

34. Van Berendoncks AM, Beckers P, Hoymans VY, et al. Exercise training reduces circulating adiponectin levels in patients with chronic heart failure. Clin Sci 2010;118:281-9.

35. Conraads VM, Beckers P, Vaes J, et al. Combined endurance/ resistance training reduces NT-proBNP levels in patients with chronic heart failure. Eur Heart J 2004;25:1797-805.

36. Yamamoto U, Mohri M, Shimada K, et al. Six-month aerobic exercise training ameliorates central sleep apnea in patients with chronic heart failure. J Card Fail 2007;13:825-9.

37. Delagardelle $\mathrm{C}$, Feiereisen $\mathrm{P}$, Vaillant $\mathrm{M}$, et al. Reverse remodelling through exercise training is more pronounced in non-ischemic heart failure. Clin Res Cardiol 2008:97:865-71.

38. Nishi I, Noguchi T, Iwanaga Y, et al. Effects of exercise training in patients with chronic heart failure and advanced left ventricular systolic dysfunction receiving $\beta$-blockers. Circ J 2011;75:1649-55.

39. Kitzman DW, Brubaker P, Morgan T, et al. Effect of Caloric Restriction or Aerobic Exercise Training on Peak Oxygen Consumption and Quality of Life in Obese Older Patients With Heart Failure With Preserved Ejection Fraction: A Randomized Clinical Trial. JAMA 2016;315:36-46.

40. Kitzman DW, Hundley WG, Brubaker PH, et al. A randomized double-blind trial of enalapril in older patients with heart failure and preserved ejection fraction: effects on exercise tolerance and arteria distensibility. Circ Heart Fail 2010;3:477-85.

41. Karavidas A, Driva M, Parissis JT, et al. Functional electrical stimulation of peripheral muscles improves endothelial function and clinical and emotional status in heart failure patients with preserved left ventricular ejection fraction. Am Heart $J$ 2013;166:760-7.

42. Palau P, Domínguez E, Núñez E, et al. Effects of inspiratory muscle training in patients with heart failure with preserved ejection fraction. Eur J Prev Cardiol 2014;21:1465-73.

43. Aksoy S, Findikoglu G, Ardic F, et al. Effect of 10-Week Supervised Moderate-Intensity Intermittent vs. Continuous Aerobic Exercise Programs on Vascular Adhesion Molecules in Patients with Heart Failure. Am J Phys Med Rehabil 2015;94:898-911.

44. Antonicelli R, Spazzafumo L, Scalvini S, et al. Exercise: a "new drug" for elderly patients with chronic heart failure. Aging 2016;8:860-72.

45. Krishna BH, Pal P, Pal GK, et al. A Randomized Controlled Trial to Study the Effect of Yoga Therapy on Cardiac Function and N Terminal Pro BNP in Heart Failure. Integr Med Insights 2014:9:IMI. S13939.
46. Jónsdóttir S, Andersen KK, Sigurosson AF, et al. The effect of physical training in chronic heart failure. Eur $J$ Heart Fail 2006;8:97-101.

47. Wisløff U, Støylen A, Loennechen JP, et al. Superior cardiovascular effect of aerobic interval training versus moderate continuous training in heart failure patients: a randomized study. Circulation 2007:115:3086-94.

48. Conraads VM, Vanderheyden M, Paelinck B, et al. The effect of endurance training on exercise capacity following cardiac resynchronization therapy in chronic heart failure patients: a pilot trial. Eur J Cardiovasc Prev Rehabil 2007;14:99-106.

49. Guazzi M, Vitelli A, Arena R. The effect of exercise training on plasma NT-pro-BNP levels and its correlation with improved exercise ventilatory efficiency in patients with heart failure. Int $\mathrm{J}$ Cardiol 2012:158:290-1.

50. Meyer T, Schwaab B, Görge G, et al. Can serum NT-proBNP detect changes of functional capacity in patients with chronic heart failure? Z Kardiol 2004;93:540-5.

51. Sandri M, Kozarez I, Adams V, et al. Age-related effects of exercise training on diastolic function in heart failure with reduced ejection fraction: the Leipzig Exercise Intervention in Chronic Heart Failure and Aging (LEICA) Diastolic Dysfunction Study. Eur Heart J 2012;33:1758-68.

52. Maria Sarullo F, Gristina T, Brusca I, et al. Effect of physical training on exercise capacity, gas exchange and $\mathrm{N}$-terminal pro-brain natriuretic peptide levels in patients with chronic heart failure. Eur $J$ Cardiovasc Prev Rehabil 2006;13:812-7.

53. Ahmad T, Fiuzat M, Mark DB, et al. The effects of exercise on cardiovascular biomarkers in patients with chronic heart failure. Am Heart J 2014;167:193-202.

54. Eleuteri E, Mezzani A, Di Stefano A, et al. Aerobic training and angiogenesis activation in patients with stable chronic heart failure: a preliminary report. Biomarkers 2013;18:418-24.

55. Nilsson BB, Westheim A, Risberg MA, et al. No effect of group-based aerobic interval training on $\mathrm{N}$-terminal pro- $\mathrm{B}$-type natriuretic peptide levels in patients with chronic heart failure. Scand Cardiovasc $J$ 2010;44:223-9.

56. Marco E, Ramírez-Sarmiento AL, Coloma A, et al. High-intensity vs. sham inspiratory muscle training in patients with chronic heart failure: a prospective randomized trial. Eur J Heart Fail 2013:15:892-901.

57. Fu TC, Wang $\mathrm{CH}$, Lin PS, et al. Aerobic interval training improves oxygen uptake efficiency by enhancing cerebral and muscular hemodynamics in patients with heart failure. Int $\mathrm{J}$ Cardiol 2013;167:41-50.

58. Butterfield JA, Faddy SC, Davidson P, et al. Exercise training in patients with stable chronic heart failure: effects on thoracic impedance cardiography and B-type natriuretic peptide. $J$ Cardiopulm Rehabil Prev 2008;28:33-7.

59. Gary RA, Cress ME, Higgins MK, et al. Combined aerobic and resistance exercise program improves task performance in patients with heart failure. Arch Phys Med Rehabil 2011;92:1371-81.

60. Malfatto G, Branzi G, Osculati G, et al. Improvement in left ventricular diastolic stiffness induced by physical training in patients with dilated cardiomyopathy. J Card Fail 2009;15:327-33.

61. Kobayashi N, Tsuruya Y, Iwasawa T, et al. Exercise training in patients with chronic heart failure improves endothelial function predominantly in the trained extremities. Circ J 2003;67:505-10.

62. Norman JF, Pozehl BJ, Duncan KA, et al. Effects of Exercise Training versus Attention on Plasma B-type Natriuretic Peptide, 6-Minute Walk Test and Quality of Life in Individuals with Heart Failure. Cardiopulm Phys Ther J 2012;23:19-25.

63. Parrinello G, Torres D, Paterna S, et al. Short-term walking physical training and changes in body hydration status, B-type natriuretic peptide and C-reactive protein levels in compensated congestive heart failure. Int J Cardiol 2010;144:97-100.

64. Stevens AL, Hansen D, Herbots L, et al. Exercise training improves insulin release during glucose tolerance testing in stable chronic heart failure patients. J Cardiopulm Rehabil Prev 2015;35:37-46.

65. Brubaker PH, Moore JB, Stewart KP, et al. Endurance exercise training in older patients with heart failure: results from a randomized, controlled, single-blind trial. J Am Geriatr Soc 2009;57:1982-9.

66. Karavidas A, Parissis J, Arapi S, et al. Effects of functional electrical stimulation on quality of life and emotional stress in patients with chronic heart failure secondary to ischaemic or idiopathic dilated cardiomyopathy: a randomised, placebo-controlled trial. Eur J Heart Fail 2008:10:709-13.

67. Kato Y, Suzuki S, Uejima T, et al. Impact of BNP level and peak VO on future heart failure events: comparison between sinus rhythm and atrial fibrillation. Heart Vessels 2017;32:428-35. 
68. Kawauchi TS, Umeda IIK, Braga LM, et al. Is there any benefit using low-intensity inspiratory and peripheral muscle training in heart failure? A randomized clinical trial. Clin Res Cardiol 2017;106:676-85.

69. Yeh GY, Wood MJ, Lorell BH, et al. Effects of tai chi mind-body movement therapy on functional status and exercise capacity in patients with chronic heart failure: a randomized controlled trial. Am J Med 2004;117:541-8.

70. Yeh GY, McCarthy EP, Wayne PM, et al. Tai chi exercise in patients with chronic heart failure: a randomized clinical trial. Arch Intern Med 2011;171:750-7.

71. Cipriano G, Cipriano VT, da Silva VZ, et al. Aerobic exercise effect on prognostic markers for systolic heart failure patients: a systematic review and meta-analysis. Heart Fail Rev 2014;19:655-67.

72. Pascual-Figal DA, Januzzi JL. The biology of ST2: the International ST2 Consensus Panel. Am J Cardiol 2015;115:3B-7.

73. Zhong $Y$, Wang R, Yan L, et al. Copeptin in heart failure: Review and meta-analysis. Clinica Chimica Acta 2017;475:36-43.

74. Balling L, Gustafsson F. Copeptin as a biomarker in heart failure. Biomark Med 2014;8:841-54.

75. Yuyun MF, Narayan HK, Ng LL. Prognostic significance of adrenomedullin in patients with heart failure and with myocardial infarction. Am J Cardiol 2015;115:986-91.

76. Felker GM, Fiuzat M, Thompson V, et al. Soluble ST2 in ambulatory patients with heart failure: Association with functional capacity and long-term outcomes. Circ Heart Fail 2013;6:1172-9.

77. Felker GM, Whellan D, Kraus WE, et al. N-terminal pro-brain natriuretic peptide and exercise capacity in chronic heart failure: data from the Heart Failure and a Controlled Trial Investigating Outcomes of Exercise Training (HF-ACTION) study. Am Heart J 2009;158:S37-44.

78. Nishikimi T, Nakagawa Y. Adrenomedullin as a Biomarker of Heart Failure. Heart Fail Clin 2018;14:49-55.

79. Tucker WJ, Lijauco CC, Hearon CM, et al. Mechanisms of the Improvement in Peak VO 2 With Exercise Training in Heart Failure With Reduced or Preserved Ejection Fraction. Heart, Lung and Circulation 2018;27:9-21.

80. Komajda M, Carson PE, Hetzel S, et al. Factors associated with outcome in heart failure with preserved ejection fraction: findings from the Irbesartan in Heart Failure with Preserved Ejection Fraction Study (I-PRESERVE). Circ Heart Fail 2011;4.

81. Masson S, Latini R, Anand IS, et al. Direct comparison of B-type natriuretic peptide (BNP) and amino-terminal proBNP in a large population of patients with chronic and symptomatic heart failure: the Valsartan Heart Failure (Val-HeFT) data. Clin Chem 2006;52:1528-38.

82. van Veldhuisen DJ, Linssen GC, Jaarsma T, et al. B-type natriuretic peptide and prognosis in heart failure patients with preserved and reduced ejection fraction. J Am Coll Cardiol 2013;61:1498-506.

83. Tromp J, Khan MA, Klip IT, et al. Biomarker Profiles in Heart Failure Patients With Preserved and Reduced Ejection Fraction. J Am Heart Assoc 2017;6:e003989.

84. Sanders-van Wijk S, van Empel V, Davarzani N, et al. Circulating biomarkers of distinct pathophysiological pathways in heart failure with preserved vs. reduced left ventricular ejection fraction. Eur $J$ Heart Fail 2015;17:1006-14.

85. Pearson MJ, Smart NA. Aerobic Training Intensity for Improved Endothelial Function in Heart Failure Patients: A Systematic Review and Meta-Analysis. Cardiol Res Pract 2017;2017:1-10.

86. Ellingsen $\varnothing$, Halle $M$, Conraads V, et al. High-Intensity Interval Training in Patients With Heart Failure With Reduced Ejection Fraction. Circulation 2017;135:839-49.

87. Salmoirago-Blotcher E, Wayne PM, Dunsiger S, et al. Tai Chi Is a Promising Exercise Option for Patients With Coronary Heart Disease Declining Cardiac Rehabilitation. J Am Heart Assoc 2017;6:e006603.

88. Liu T, Chan AW, Liu YH, et al. Effects of Tai Chi-based cardiac rehabilitation on aerobic endurance, psychosocial well-being, and cardiovascular risk reduction among patients with coronary heart disease: A systematic review and meta-analysis. Eur J Cardiovasc Nurs 2018;17:1474515117749592.

89. Adamopoulos S, Schmid J-P, Dendale P, et al. Combined aerobic/ inspiratory muscle training vs. aerobic training in patients with chronic heart failure. Eur J Heart Fail 2014;16:574-82.

90. Caminiti G, Volterrani M, Marazzi G, et al. Tai chi enhances the effects of endurance training in the rehabilitation of elderly patients with chronic heart failure. Rehabil Res Pract 2011;2011:1-6.

91. Jiao D-D, Zhang W-Y, Xu J, et al. Effect of weight support exercise therapy on the cardiac function in patients with chronic heart failure. $J$ Hainan Med Univ 2016;22:21-3.

92. Schoenrath F, Markendorf S, Brauchlin AE, et al. Robot-assisted training for heart failure patients - a small pilot study. Acta Cardiol 2015;70:665-71.

93. Ibrahim NE, Gaggin HK, Konstam MA, et al. Established and Emerging Roles of Biomarkers in Heart Failure Clinical Trials. Circ Heart Fail 2016;9:e002528.

94. Meijers WC, van der Velde AR, Muller Kobold AC, et al. Variability of biomarkers in patients with chronic heart failure and healthy controls. Eur J Heart Fail 2017; 19:357-65. 